\title{
ON SUPERCUSPIDAL REPRESENTATIONS OF THE METAPLECTIC GROUP
}

BY

\author{
JAMES MEISTER
}

\begin{abstract}
The Weil representations associated to anisotropic quadratic forms in one and three variables are used to study supercuspidal representations of the two-fold metaplectic covering group $\overline{\mathrm{GL}}_{2}(k)$, where $k$ is a local nonarchimedean field of odd residual characteristic. The principal result is the explicit calculation of certain Whittaker functionals for any square-integrable irreducible admissible genuine representation of $\overline{\mathrm{GL}}_{2}(k)$. In particular, a recent conjecture of Gelbart and Piatetski-Shapiro is answered by obtaining a bijection between the set of quasicharacters of $k^{*}$ and the set of irreducible admissible genuine distinguished representations of $\overline{\mathrm{GL}}_{2}(k)$, i.e. those representations possessing only one Whittaker functional, or, equivalently, those having a unique Whittaker model. The distinguished representations are precisely the representations attached to the Weil representation associated to a one dimensional form.

The local piece of the generalized Shimura correspondence between automorphic forms of $\overline{G L}_{2}(A)$ and $G_{2}(A)$ is also treated. Based upon a conjecture of the equivalences among the constituents of the Weil representations associated to two nonequivalent ternary forms, evidence for the explicit form of the local piece of this global correspondence, restricted to supercuspidal representations of $\overline{\mathrm{GL}}_{2}(k)$, is presented. In this form, the map is shown to be injective and its image is described.
\end{abstract}

0. Introduction. A fundamental result in the theory of representations of $\mathrm{GL}_{2}$ over a local field $k$ is the "Existence. and Uniqueness of Whittaker Models". That is, fixing a nontrivial additive character $\psi$ of $k$ ( $k$ nonarchimedean), for an irreducible admissible infinite dimensional representation $\pi$, there is, in the space $W(\pi, \psi)$ of locally constant functions on $\mathrm{GL}_{2}(k)$ satisfying

$$
W\left[\left(\begin{array}{ll}
1 & x \\
0 & 1
\end{array}\right) g\right]=\psi(x) W(g), \quad x \in k,
$$

a unique subspace which as a right $\mathrm{GL}_{2}(k)$ module is equivalent to $\pi$. It is easy to see that this is equivalent to the statement that the space of linear functionals $l$ on the representation space $V_{\pi}$ satisfying

$$
l\left[\pi\left(\begin{array}{cc}
1 & x \\
0 & 1
\end{array}\right) v\right]=\psi(x) l(v), \quad x \in k, v \in V_{\pi}
$$

has dimension equal to one.

Received by the editors September 25, 1979 and, in revised form, May 25, 1980.

AMS (MOS) subject classifications (1970). Primary 22E50.

Key words and phrases. Metaplectic group, supercuspidal representations, Whittaker model, Shimura correspondence. 
For an arbitrary irreducible admissible infinite dimensional representation of the metaplectic two-fold covering group $\overline{\mathrm{GL}}_{2}(k)$, this is no longer true. Those representations having a unique linear functional (up to a constant) satisfying (0.1) are called distinguished, and in fact for genuine representations (i.e. those nontrivial on the square roots of unity), these are few in number. We show, for odd residual characteristic, that the only irreducible admissible genuine distinguished representations of $\overline{\mathrm{GL}}_{2}(k)$ are those attached to the Weil representation associated to a one dimensional quadratic form and that these are in 1-1 correspondence with the set of quasi-characters of $k^{*}$.

There are global analogues of the above statements that have importance in the theory of modular forms of half-integral weight. Here, the notion of a distinguished representation generalizes the idea of a cusp form being determined by its first Fourier coefficient. Hence these global results imply that a modular form of half-integral weight whose coefficients $a_{n}$ vanish for $n \neq m t^{2}, t$ a square-free integer, is a translate of the theta form $\theta_{\chi}(z)$. A complete discussion of these global results appears in [5].

Gelbart and Piatetski-Shapiro have attached to each irreducible admissible representation $\pi$ of $\overline{\mathrm{GL}}_{2}(k)$ a finite (and if $\pi$ is infinite dimensional, nonempty) set $\Omega_{\psi}(\pi)$ which can be described as the set of characters $\mu$ of $\bar{Z}$ ( $Z=$ center of $\mathrm{GL}_{2}(k)$ ), extending the central character of $\pi$, such that there exists a nonzero linear functional $l$ on $V_{\pi}$ satisfying $(0.1)$ and

$$
l(\pi(\bar{z}) v)=\mu(\bar{z}) l(v), \quad \bar{z} \in \bar{Z}, v \in V_{\pi} .
$$

It is this set which is the object under investigation here. Moreover, the results in [7] imply such an $l$ is unique up to a constant. Thus the cardinality of $\Omega_{\psi}(\pi)$ equals the dimension of the set of linear functionals satisfying $(0.1)$, or is at most the number of distinct subspaces of $W(\pi, \psi)$ realizing $\pi$. Our main theorem calculates $\Omega_{\psi}(\pi)$ explicitly for any square-integrable irreducible admissible genuine representation of $\overline{\mathrm{GL}}_{2}(k)$. The proof relies heavily upon the theorems and techniques in [14] and is based upon a relationship between the way an irreducible representation of the orthogonal group of an anisotropic ternary quadratic form sits inside the regular representation, and the existence of linear functionals on the space of the associated representation of $\overline{\mathrm{GL}}_{2}(k)$ satisfying $(0.1)$ and $(0.2)$.

In $\S 1$ the basic properties of representations of the orthogonal groups in one and three dimensions are collected. In $\$ 2$ we review the construction of square-integrable irreducible genuine representations of $\overline{\mathrm{SL}}_{2}(k)$ as Weil representations and extend these to $\overline{\mathrm{GL}}_{2}(k)$. A more convenient representation space is then constructed. Assuming the technical result of $\S 4$, that the constructions in $\$ 2$ exhaust the set of square-integrable irreducible admissible genuine representations of $\overline{\mathrm{GL}}_{2}(k)$, we compute $\Omega_{\psi}(\pi)$ in $\S 3$.

A second and more difficult problem is the explicit realization of the local piece of the generalized Shimura correspondence between automorphic forms of $\overline{\mathrm{GL}}_{2}$ and automorphic forms of $\mathrm{GL}_{2}$ discussed in [6]. This has been done for the principal series; in $\$ 5$ we conjecture, and provide evidence for, the form of this 
correspondence in the square-integrable case. The essential ingredient needed to complete this analysis would be a precise description of the equivalences among the constituents of the Weil representations associated to two nonequivalent ternary forms.

1. Spherical representations of an orthogonal group. Let $k$ be a nonarchimedean local field of odd residual characteristic and $\tilde{\omega}$ a local uniformizing parameter. We denote the Hilbert symbol on $k^{*} \times k^{*}$ by $(\cdot, \cdot)$ and let $\varepsilon$ be a unit in the ring of integers $O_{k}$ such that $(\varepsilon, \tilde{\omega})=-1$. Then $\Sigma=\{1, \varepsilon, \tilde{\omega}, \varepsilon \tilde{\omega}\}$ is a set of representatives for $k^{*} / k^{* 2}$.

Suppose $Q$ is an anisotropic quadratic form on the $k$-space $E$. Although many of the results in this section hold for arbitrary $(Q, E)$, we will be particularly interested in the cases where $\operatorname{dim}_{k} E$ is one or three.

First, define $q_{1}$ on $k$ by $q_{1}(X)=X^{2}, X \in k$.

Now let $H$ be the unique quaternion algebra over $k$ whose typical element is of the form $a_{0}+a_{1} \vec{i}+a_{2} \vec{j}+a_{3} \vec{k}, a_{i} \in k$, where $\vec{i}^{2}=\varepsilon, \vec{j}^{2}=\tilde{\omega}, \vec{k}^{2}=-\varepsilon \tilde{\omega}$, and let $H_{0}$ be the space of pure quaternions. Define $q_{3}$ on $H_{0}$ by

$$
q_{3}(X)=X \bar{X}, \quad X \in H_{0} ;
$$

that is, the norm of $X$.

The basic facts about these forms are contained in the following lemma.

LEMMA 1.1. (a) Up to equivalence there are four one dimensional (respectively three dimensional) anisotropic quadratic forms; namely $a q_{1}$ (respectively $a q_{3}$ ), where $a \in \Sigma$.

(b) If $\mathrm{H}_{0}^{*}$ denotes $\mathrm{H}_{0}-\{0\}$, then

$$
a q_{3}\left(H_{0}^{*}\right)=\left\{t \in k^{*}:-a^{-1} t \notin k^{* 2}\right\} .
$$

Proof. The assertions involving $q_{1}$ are trivial. Those involving $q_{3}$ follow easily from well-known properties of quadratic forms (see [15]).

Let $O(Q)$ denote the orthogonal group of $(Q, E)$ and $\nu$ the regular representation of $O(Q)$ acting on $L^{2}(E)$ :

$$
\nu(g) f(X)=f\left(g^{-1} X\right), \quad g \in O(Q), X \in E .
$$

We say a representation $\tau$ of $O(Q)$ is spherical if it is contained in $\nu$. Similar definitions are made when $O(Q)$ is replaced by $O^{+}(Q)=\{g \in O(Q)$ : $\operatorname{det} g=1\}$.

Observe that if $E^{*}=E-\{0\}$, then $Q\left(E^{*}\right)$ is a union of $k^{* 2}$ cosets in $k^{*}$. If we set $E_{b}=\left\{X \in E: Q(X) \in b k^{* 2}\right\}, b \in k^{*}$, then

$$
L^{2}(E)=\bigoplus_{b \in B} L^{2}\left(E_{b}\right)
$$

where $B$ is a complete set of $k^{* 2}$ coset representatives in $Q\left(E^{*}\right)$. Moreover each summand of this orthogonal direct sum is invariant under $\nu$. This leads to the following definition.

DefinITION 1.2. Suppose $\tau$ is an irreducible spherical representation of $O(Q)$. Define $C_{Q, \tau}$ to be the set of those cosets $b k^{* 2}$ (in $Q\left(E^{*}\right)$ ) such that $\tau$ is contained in the restriction of $\nu$ to $L^{2}\left(E_{b}\right)$. 
As shown in [14] we have:

LEMMA 1.3. Suppose the irreducible spherical representation $\tau$ acts in the space $V_{\tau}$. Fix $X$ in $E^{*}$ and set

$$
O(Q)_{X}=\{g \in O(Q): g X=X\} .
$$

Then $Q(X) k^{* 2}$ is in $C_{Q, \tau}$ if and only if there is a nonzero vector $v_{X}$ in $V_{\tau}$ invariant under $O(Q)_{X}$. Moreover, if such a vector exists, it is unique up to a scalar.

We wish now to calculate $C_{Q, \tau}$ for $Q=q_{3}$. The following lemma explains why the study of representations of $O\left(q_{3}\right)$ is essentially reduced to the study of representations of $H^{*} / k^{*}$.

LEMMA 1.4. (a) $O^{+}\left(c q_{3}\right) \simeq H^{*} / k^{*}$.

(b) $O\left(c q_{3}\right) \simeq O^{+}\left(c q_{3}\right) \times \mathbf{Z}_{2}$.

Proof. See [14].

Suppose $\sigma$ is an irreducible one dimensional representation of $H^{*} / k^{*}$. It is a corollary of Lemma 4.1 of [9] that $\sigma$ is of the form $\sigma(h)=\chi(h \bar{h})$, where $\chi$ is a quasi-character of $k^{*}$. Moreover $\sigma$ trivial on $k^{*}$ implies $\chi$ is trivial on $k^{* 2}$, i.e., $\chi$ is of the form $\chi_{b}(t)=(b, t), b \in \Sigma$. We will denote such a $\sigma$ simply as $\chi_{b}$ when convenient.

If $\operatorname{dim} \sigma>1$ it is known that one can attach to $\sigma$ a nontrivial element of $\Sigma$, denoted $\alpha(\sigma)$, in the manner outlined in the following remark.

Remark 1.5. Let $L=\left\{X \in H:|X \bar{X}|_{k}=1\right\}$ and let $P L$ denote the quotient group of $L$ by its center. Then $P L$ is a normal subgroup of index 2 in $H^{*} / k^{*}$. In [8], all of the irreducible representations of $P L$ are constructed and to each a nontrivial element of $\Sigma$ is associated in the following manner.

Let $U_{e}^{*}, U_{\tilde{\omega}}^{*}$, and $U_{e \tilde{\omega}}^{*}$ be subgroups of $L$ consisting of nonzero elements $a+b \vec{i}$, $a+b \vec{j}$, and $a+b \vec{k}^{\prime}$ respectively (here $\vec{k}^{\prime}$ is chosen such that $\left.q_{3}\left(\overrightarrow{k^{\prime}}\right)=\varepsilon \tilde{\omega}\right)$. Note that in each class of conjugate elements of $L$ there is an element belonging to one of these subgroups. Given an irreducible representation $T$ of $L$, trivial on its center, the trace of $T(X)$ is nonzero on only one of these subgroups, providing that $|X-\bar{X}|_{k}>q^{-r}$, for an integer $r$ depending on $T\left(q=|\tilde{\omega}|^{-1}\right)$. Define $\alpha(T)$ to be the element of $\Sigma$ such that this distinguished subgroup is $U_{\alpha(T)}^{*}$.

Now let $\sigma$ be an irreducible representation of $H^{*} / k^{*}$. Upon restriction to $P L, \sigma$ is either irreducible or the direct sum of two conjugate irreducibles. By its definition it is clear that if $T$ and $T^{\prime}$ are conjugate irreducibles of $P L$, then $\alpha(T)=\alpha\left(T^{\prime}\right)$. We therefore set $\alpha(\sigma)=\alpha(T)$ if $\left.\sigma\right|_{P L}=T$ or if, say, $\left.\sigma\right|_{P L}=T \oplus T^{\prime}$.

Finally, consider $O\left(c q_{1}\right) \simeq \mathbf{Z}_{2}$. We label the two irreducible (spherical) representations of $\mathbf{Z}_{2}$ by $\theta_{1}$ and $\theta_{-1}$, where $\theta_{i}(-1)=i, i=1,-1$.

THEOREM 1.6. (1) $C_{c q_{1}, \theta_{1}}=C_{c q_{1}, \theta_{-1}}=\left\{c k^{* 2}\right\}$.

(2) Suppose $\sigma$ is any irreducible representation of $H^{*} / k^{*}$. Then $\sigma$ extends in a unique manner to a spherical representation of $O\left(c q_{3}\right)$ denoted by $\tilde{\sigma}$. Moreover,

(a) $C_{c q_{3}, \tilde{x}_{b}}=\left\{t k^{* 2}:-c^{-1} t \notin k^{* 2}\right\}$ if $b \in k^{* 2}$; 
(b) $C_{c q_{3}, \tilde{x}_{b}}=\left\{-c b k^{* 2}\right\}$ if $b \notin k^{* 2}$;

(c) $C_{c q_{3}, \tilde{\sigma}}=\left\{c e_{1} k^{* 2}, c e_{2} k^{* 2}\right\}$ if $\operatorname{dim} \sigma>1$,

where $e_{1}$ and $e_{2}$ are two $k^{* 2}$ coset representatives in $q_{3}\left(H_{0}^{*}\right)$ such that $\left(\alpha(\sigma),-e_{1}\right)=$ $\left(\alpha(\sigma),-e_{2}\right)=-1$.

Proof. (1) Obvious since $c q_{1}\left(k^{*}\right)=c k^{* 2}$.

(2) By Lemma 1.3 we need only consider the case where $c=1$; indeed if $C_{Q, \tau}=\left\{s_{i} k^{* 2}: i \in I\right\}$, then $C_{c Q, \tau}=\left\{c s_{i} k^{* 2}: i \in I\right\}$.

The proof of (2) is established in two steps. The first step is to consider $\nu^{+}=\left.\nu\right|_{O^{+}\left(q_{3}\right)}$ and to determine those $b$ in $\Sigma$ such that a given irreducible spherical representation $\sigma$ of $\mathrm{O}^{+}\left(q_{3}\right)$ is contained in $\left.\nu^{+}\right|_{L^{2}\left(H_{0_{b}}\right)}$. This is done in [14], but we sketch the arguments here. The second step is to show that an irreducible spherical representation $\sigma$ of $\mathrm{O}^{+}\left(q_{3}\right)$ extends uniquely to an irreducible spherical representation $\tilde{\boldsymbol{\sigma}}$ of $O\left(q_{3}\right)$. This, then, implies that $C_{q_{3}, \tilde{\sigma}}$ consists of those cosets $b k^{* 2}$ such that $\sigma$ is contained in $\left.v^{+}\right|_{L^{2}\left(H_{0_{b}}\right)}$.

Now $O^{+}\left(q_{3}\right)$ acts transitively on $\left\{X \in H_{0}: q_{3}(X)=t\right\}$, any $t \in k$, and it is not hard to show that the analogue of Lemma 1.3 holds for $\nu^{+}$, i.e., $\sigma$ is contained in $\left.\nu^{+}\right|_{L^{2}\left(H_{0 q_{3}(X)}\right)}$ if and only if there is a nonzero vector $v_{X}$ fixed by $O^{+}\left(q_{3}\right)_{X}$. Fix $X$ in $H_{0}^{*}$ and consider $S_{X}=\left\{h \in H^{*}: h X=X h\right\} . S_{X}$ is isomorphic to the multiplicative group of the quadratic extension $k\left(\sqrt{-q_{3}(X)}\right)$. (Note $-q_{3}(X) \notin k^{* 2}$ by Lemma 1.1(b).) If we denote the image of $S_{X}$ under the projection $H^{*} \rightarrow H^{*} / k^{*}$ by $T_{X}$, we see that the torus $T_{X}$ is isomorphic to $O^{+}\left(q_{3}\right)_{X}$.

Consider $\sigma \equiv 1$, the trivial representation of $H^{*} / k^{*}$, and fix $X$ in $H_{0}^{*}$. Obviously $\left.\sigma\right|_{T_{X}} \equiv 1$, so $\sigma$ is contained in $L^{2}\left(H_{0_{a_{3}(X)}}\right)$. That is, $\sigma$ is contained in $L^{2}\left(H_{0_{b}}\right)$ for any $b \in q_{3}\left(H_{0}^{*}\right)$ (i.e., three $L^{2}$ spaces).

Consider $\sigma=\chi_{b}$. Then $\left.\chi_{b}\right|_{T_{X}} \equiv 1$ if and only if $(b, h \bar{h})=1$ for all $h \in T_{X}$, that is, if and only if $-q_{3}(X) k^{* 2}=b k^{* 2}$, i.e., $q_{3}(X) \in-b k^{* 2}$. Thus $\sigma$ is contained in exactly one space: $L^{2}\left(H_{0_{-}}\right)$.

Finally consider the case when $\operatorname{dim} \sigma>1$. We have $-q_{3}\left(H_{0}^{*}\right)=\left\{\varepsilon k^{* 2}, \tilde{\omega} k^{* 2}\right.$, $\left.\varepsilon \tilde{\omega} k^{* 2}\right\}$ and since $(\alpha(\sigma), \varepsilon \tilde{\omega})=(\alpha(\sigma), \varepsilon)(\alpha(\sigma), \tilde{\omega})$ it is clear that there are exactly two (nonequivalent) $k^{* 2}$ coset representatives $e_{1}, e_{2}$ in $q_{3}\left(H_{0}^{*}\right)$ such that $\left(\alpha(\sigma),-e_{1}\right)=$ $\left(\alpha(\sigma),-e_{2}\right)=-1$. To compute when tori $T_{X}$ have the property that $\left.\sigma\right|_{T_{X}}$ contains the trivial representation (i.e., fixes a vector under $\left.O^{+}\left(q_{3}\right)_{X}\right)$, we appeal to Theorem 2.5 of [14] which implies that they are exactly those such that $\left(\alpha(\sigma),-q_{3}(X)\right)=-1$. This shows, incidentally, that every irreducible representation of $H^{*} / k^{*}$ is spherical.

The proof of Theorem 1.4 will be complete as soon as we show the following:

Proposition 1.7. Suppose $\sigma$ is an irreducible representation of $H^{*} / k^{*}$. Then $\sigma$ extends uniquely to a spherical representation of $O\left(q_{3}\right)$.

Proof. Let $\theta$ denote an irreducible representation of $\mathbf{Z}_{2}$, i.e., $\theta=\theta_{1}$ or $\theta_{-1}$. Then $\sigma \otimes \theta$ is an irreducible representation of $O\left(q_{3}\right)$ and if $\sigma \otimes \theta$ is spherical, we will show $\theta$ is uniquely determined. Note $\sigma \otimes \theta$ must be spherical for at least one $\theta$ since $\sigma$ is spherical. 
Consider first the case $\sigma=\chi_{b}, b \notin k^{* 2}$, and assume $\sigma \otimes \theta$ is spherical. Thus $\sigma \otimes \theta$ is contained only in $L^{2}\left(H_{0_{-b}}\right)$ since $\sigma$ is contained only in $L^{2}\left(H_{0_{-b}}\right)$. Thus, by Lemma 1.3, $\sigma \otimes \theta$ must have a nonzero vector $v_{X}$ invariant by $O\left(q_{3}\right)_{X}$ if $q_{3}(X) k^{* 2}$ $=-b k^{* 2}$. Fix such an $X$ and choose $g \in O_{X}$ with $\operatorname{det} g=-1$. Then $g X=X$ implies $-g X=-X$ and, of course, $-g \in O^{+}\left(q_{3}\right)$. (If $g^{\prime} \in O_{X}$, det $g^{\prime}=-1$ then $g^{\prime}=g s$ with $s \in O_{X}^{+}$and we will obtain the same thing.) So $-g$ corresponds to an $h \in H^{*}$ with $h X h^{-1}=-X$. Then

$$
v_{X}=\sigma \otimes \theta(g) v_{X}=\sigma \otimes \theta(-(-g)) v_{X}=\theta(-1) \sigma(h) v_{X}
$$

and so $\theta(-1)$ is determined uniquely. Furthermore, a different choice $Y$ in $H_{0}^{*}$ such that $q_{3}(Y) k^{* 2}=-b k^{* 2}$ yields exactly (1.1) again. Indeed, if $Y=a X$, choose the same $g \in O_{X}$, det $g=-1$ as before. If $q_{3}(Y)=q_{3}(X)$, then $Y=k X k^{-1}$ for some $k \in H^{*}$ and if $h X h^{-1}=-X$, set $-g=k h k^{-1}$. Then $g \in O_{Y}$, det $g=-1$, and since $v_{Y}=\sigma(k) v_{X}$ (we will explain this in $\S 2$ ),

$$
\sigma(k) v_{X}=\theta(-1) \sigma\left(k h k^{-1}\right) \sigma(k) v_{X}
$$

which is exactly (1.1). Thus $\sigma=\chi_{b}$ extends uniquely to a spherical representation of $O\left(q_{3}\right)$.

Consider now the case when $\operatorname{dim} \sigma>1$. We know $\sigma$ lives in exactly two $L^{2}$ spaces, $L^{2}\left(H_{0_{b_{1}}}\right)$ and $L^{2}\left(H_{0_{b_{2}}}\right)$. Suppose $\theta$ and $\theta^{\prime}$ are two irreducible representations of $\mathbf{Z}_{2}$ such that $\sigma \otimes \theta$ lives in $L^{2}\left(H_{0_{b_{1}}}\right)$ and $\sigma \otimes \theta^{\prime}$ lives in $L^{2}\left(H_{0_{b}}\right)$. We want to show $\theta=\theta^{\prime}$. Choose $X_{1}$ and $X_{2}$ in $H_{0}^{*}$ such that $q_{3}\left(X_{i}\right) k^{* 2}=b_{i} k^{* 2}, i=1,2$. Without loss of generality we may assume

$$
\left(v_{X_{1}}, v_{X_{2}}\right) \neq 0
$$

where the inner product on the representation space is chosen so that $\sigma$ is unitary; indeed $\sigma$ irreducible implies there is a $g \in O^{+}\left(q_{3}\right)$ such that $\left(\sigma(g) v_{X_{1}}, v_{X_{2}}\right)=$ $\left(v_{g X_{1}}, v_{X_{2}}\right) \neq 0$. Arguing as above, we are led to two equations:

$$
\begin{gathered}
\theta(-1) \sigma\left(h_{1}\right) v_{X_{1}}=v_{X_{1}}, \quad \text { where } h_{1} X_{1} h_{1}^{-1}=-X_{1}, \\
\theta^{\prime}(-1) \sigma\left(h_{2}\right) v_{X_{2}}=v_{X_{2}}, \quad \text { where } h_{2} X_{2} h_{2}^{-1}=-X_{2} .
\end{gathered}
$$

Suppose we could arrange to take $h_{1}=h_{2}=h$. Then $\theta(-1)=\theta^{\prime}(-1)$; otherwise $-\left(v_{X_{1}}, v_{X_{2}}\right)=\left(\sigma(h) v_{X_{1}}, \sigma(h) v_{X_{2}}\right)=\left(v_{X_{1}}, v_{X_{2}}\right)$ contradicting (1.2). That we can arrange this is a consequence of the lemma below and we see $\sigma$ extends uniquely to a spherical representation of $O\left(q_{3}\right)$.

Finally, using these methods, it is clear that the trivial representation $\sigma \equiv 1$ extends to a spherical representation of $O\left(q_{3}\right)$ as $\sigma \otimes \theta_{1}$.

Lemma 1.8. Suppose $X$ and $Y$ are in $H_{0}^{*}$. Then there is an $h \in H^{*}$ such that $h X h^{-1}=-X$ and $h Y h^{-1}=-Y$.

Proof. In view of Lemma 1.4(a), it suffices to produce a $g \in O^{+}\left(q_{3}\right)$ such that $g X=-X$ and $g Y=-Y$. The lemma is clear if $X$ and $Y$ are linearly dependent so assume otherwise. Let $W$ be the space spanned by $X$ and $Y$ and $W^{\perp}$ be the 
orthogonal complement. Define a linear transformation $g: H_{0} \rightarrow H_{0}$ by $\left.g\right|_{W}=-I$, $\left.g\right|_{W^{\perp}}=+I$. Then $g \in O^{+}\left(q_{3}\right)$ and certainly $g X=-X$ and $g Y=-Y$.

With this, the proof of Theorem 1.6 is complete.

There are some corollaries to Proposition 1.7 that will be useful in later sections.

Corollary 1.9. Suppose $b \notin k^{* 2}$. Then $\chi_{b}$ extends to the spherical representation $\chi_{b} \otimes \theta_{i}$ where $i=-(b,-1) \in \mathbf{Z}_{2}\left(\right.$ recall $\left.\theta_{k}(-1)=k, k=1,-1\right)$.

Proof. We do this by cases. If $b=\varepsilon$, take, in the notation of Proposition 1.7, $X=\vec{i}$, since $q_{3}(\vec{i})=-\varepsilon$. Then let $h=\vec{j} ; h \overrightarrow{i h}^{-1}=\vec{j} \overrightarrow{i j}^{-1}=-\vec{i}$. Equation (1.1) reads

$$
1=\theta(-1) \chi_{e}(\vec{j}),
$$

that is, $1=\theta(-1)(\varepsilon,-\tilde{\omega})$, so $\theta(-1)=(\varepsilon,-\tilde{\omega})=(\varepsilon, \tilde{\omega})=-1$ as expected.

For $b=\tilde{\omega}$ take $X=\vec{j}$ and $h=\vec{i}$. Equation (1.1) gives

$$
1=\theta(-1)(\tilde{\omega},-\varepsilon)=\theta(-1)(\tilde{\omega},-1)(\tilde{\omega}, \varepsilon),
$$

so $\theta(-1)=-(\tilde{\omega},-1)$.

Finally, for $b=\varepsilon \tilde{\omega}$ take $X$ of the form $a \vec{j}+b \vec{k}$ (so that $q_{3}(X)=-\varepsilon \tilde{\omega}$ ), and taking $h=\vec{i}$ we obtain

$$
1=\theta(-1)(\varepsilon \tilde{\omega},-\varepsilon)=\theta(-1)(\varepsilon \tilde{\omega},-1)(\varepsilon, \varepsilon)(\tilde{\omega}, \varepsilon) .
$$

So $\theta(-1)=-(\varepsilon \tilde{\omega},-1)$ (the Hilbert symbol being trivial on the units).

Corollary 1.10. Suppose $\sigma$ is an irreducible representation of $H^{*} / k^{*}$ with $\operatorname{dim} \sigma>1$. Set $\alpha=\alpha(\sigma)$ and suppose $\sigma$ extends spherically to $O\left(q_{3}\right)$ as $\sigma \otimes \theta_{m}$ $(m \in\{1,-1\})$. Then $\chi_{b} \otimes \sigma$ extends spherically to $\left(\chi_{b} \otimes \sigma\right) \otimes \theta_{n}$ where:

(1) if $-1 \in k^{* 2}, n=(b, \alpha) m$.

(2) if $-1 \notin k^{* 2}$,

$$
\begin{array}{ll}
n=(b,-\varepsilon) m & \text { if } \alpha=\varepsilon \\
n=(b, \varepsilon \tilde{\omega}) m & \text { if } \alpha=\tilde{\omega} \\
n=(b, \tilde{\omega}) m & \text { if } \alpha=\varepsilon \tilde{\omega} .
\end{array}
$$

Proof. One proceeds as in Corollary 1.10; that is, by choosing convenient representatives for the various tori. We omit the repetitive details.

Definition 1.11. Suppose $Q$ is any anisotropic form and $\tau$ is an irreducible spherical representation of $O(Q)$. Define

$$
\hat{k}_{\tau}^{*}=\left\{\text { quasi-characters } \lambda \text { of } k^{*}: \tau(-I)=\lambda(-1) I\right\} .
$$

Returning to the specific case where $\sigma$ is an irreducible representation of $H^{*} / k^{*}$, $\operatorname{dim} \sigma>1$, with unique spherical extension $\tilde{\sigma}$, we have:

Corollary 1.12. Suppose $\lambda \in \hat{k}_{\sigma}^{*}$. Then $\chi_{b} \lambda \in \hat{k}_{\chi_{b}}^{*} \tilde{\otimes}_{\sigma}$ if and only if $b \in k^{* 2}$ or $b \in e_{1} e_{2} k^{* 2}$ where $e_{1}$ and $e_{2}$ are two $k^{* 2}$ coset representatives in $q_{3}\left(H_{0}^{*}\right)$ such that $\left(\alpha(\sigma),-e_{1}\right)=\left(\alpha(\sigma),-e_{2}\right)=-1$.

Proof. $\chi_{b} \lambda \in \hat{k}_{\chi_{b}}^{*} \tilde{\otimes}_{\sigma}$ if and only if $(b,-1)=(b, c)$ for a $c$ as given by Corollary 1.10. With the aid of the following table, we verify that in all six cases the only $b$ such that $(b,-1)=(b, c)$ are those congruent to 1 or $e_{1} e_{2}$. 


\begin{tabular}{|c|c|c|c|c|}
\hline$-1 \in k^{* 2}$ & $(b,-1)$ & $\begin{aligned} & \alpha(\sigma)=\varepsilon \\
& e_{1}=\tilde{\omega} \\
& e_{2}=\varepsilon \tilde{\omega} \\
& c=\varepsilon \\
&(b, c)\end{aligned}$ & $\begin{aligned} & \alpha(\sigma)=\tilde{\omega} \\
& e_{1}=\varepsilon \\
& e_{2}=\varepsilon \tilde{\omega} \\
& c=\tilde{\omega} \\
&(b, c)\end{aligned}$ & $\begin{aligned} & \alpha(\sigma)=\varepsilon \tilde{\omega} \\
& e_{1}=\tilde{\omega} \\
& e_{2}=\varepsilon \\
& c=\varepsilon \tilde{\omega} \\
&(b, c)\end{aligned}$ \\
\hline 1 & 1 & 1 & 1 & 1 \\
\hline$\varepsilon$ & 1 & 1 & -1 & -1 \\
\hline$\tilde{\omega}$ & 1 & -1 & 1 & -1 \\
\hline$\varepsilon \tilde{\omega}$ & 1 & -1 & -1 & 1 \\
\hline$-1 \notin k^{* 2}:$ & & $\begin{aligned} \alpha(\sigma) & =\varepsilon \\
e_{1} & =-\tilde{\omega} \\
e_{2} & =-\varepsilon \tilde{\omega} \\
c & =-\varepsilon\end{aligned}$ & $\begin{aligned} \alpha(\sigma) & =\tilde{\omega} \\
e_{1} & =-\tilde{\omega} \\
e_{2} & =-\varepsilon \\
c & =\varepsilon \tilde{\omega}\end{aligned}$ & $\begin{aligned} \alpha(\sigma) & =\varepsilon \tilde{\omega} \\
e_{1} & =-\varepsilon \\
e_{2} & =-\varepsilon \tilde{\omega} \\
c & =\tilde{\omega}\end{aligned}$ \\
\hline$b$ & $(b,-1)$ & $(b, c)$ & $(b, c)$ & $(b, c)$ \\
\hline 1 & 1 & 1 & 1 & 1 \\
\hline$\varepsilon$ & 1 & 1 & -1 & -1 \\
\hline$\tilde{\omega}$ & -1 & 1 & 1 & -1 \\
\hline$\varepsilon \tilde{\omega}$ & -1 & 1 & -1 & 1 \\
\hline
\end{tabular}

TABLE 1. Representation data for $\sigma$

2. Weil representations. Let $G=\mathrm{GL}_{2}(k)$ and let $\bar{G}$ be the two-fold metaplectic covering group of $G$ which we realize as the set of pairs $(g, \zeta), g \in G, \zeta \in \mathbf{Z}_{2}$, with multiplication given by the formula

$$
\left(g_{1}, \zeta_{1}\right)\left(g_{2}, \zeta_{2}\right)=\left(g_{1} g_{2}, \beta\left(g_{1}, g_{2}\right) \zeta_{1} \zeta_{2}\right) \text {. }
$$

We take $\beta$ to be the nontrivial two-cocycle on $G$ discussed in [3]. We will frequently abbreviate an element $(g, 1) \in \bar{G}$ simply as $g$.

We turn now to the construction of supercuspidal representations of $\bar{G}$. $\psi$ will denote a nontrivial additive character of $k$. If $t \in k^{*}$, denote by $\psi_{t}$ the character $\psi_{t}(x)=\psi(t x)$.

Fix a Haar measure dy (dependent on $\psi$ ) such that the Fourier transform $\hat{f}(x)=\int_{k} f(y) \psi(2 x y) d y$ satisfies the condition $\hat{f}(-x)=f(x)$. For $t \in k^{*}$, define

$$
\alpha_{\psi}(t)=\lim _{m \rightarrow \infty} \int_{P^{-m}} \psi_{t}\left(y^{2}\right) d_{t} y
$$

where $P$ denotes the $O_{k}$-ideal $(\tilde{\omega})$ and $d_{t} y$ is the corresponding Haar measure normalized as above for $\psi_{t}$. Note, by Weil, this limit exists and is an eighth root of unity.

Suppose $(Q, E)$ is an anisotropic quadratic form, say $Q \sim a_{1} X_{1}^{2}+a_{2} X_{2}^{2}$ $+\cdots+a_{n} X_{n}^{2}$. Then set, for $t \in k^{*}$,

$$
\gamma_{\psi}(t Q)=\prod_{i=1}^{n} \alpha_{\psi}\left(t a_{i}\right) .
$$


Note $\gamma_{\psi}(t Q)=\gamma_{\psi_{t}}(1 Q)$. Then $\left|\gamma_{\psi}(t Q)\right|=1$ and $\gamma_{\psi}$ has the property that for $f$ and $\hat{f}$ integrable on $E$,

$$
\int_{E} f(X) \psi(t Q(X)) d X=\gamma_{\psi}(t Q)|t|^{-n / 2} \int_{E} \hat{f}(X) \psi\left(-t^{-1} Q(X)\right) d X .
$$

Moreover,

$$
\alpha_{\psi}(a) \alpha_{\psi}(b)=\alpha_{\psi}(a b) \alpha_{\psi}(1)(a, b), \quad a, b \in k^{*}
$$

and

$$
\alpha_{\psi}\left(a^{2}\right)=\alpha_{\psi}(1)
$$

For more details, see $\S 1$ of [13].

Lemma 2.1. Suppose $Q=c q_{1}$ or $c q_{3}$. Then

$$
\gamma_{\psi}(a Q) \gamma_{\psi}^{-1}(t a Q)=\gamma_{\psi}(Q) \gamma_{\psi}^{-1}(t Q)
$$

for all $t \in k^{*}$ if and only if $a \in k^{* 2}$.

Proof. Take $c=1$ (similar proof otherwise) and suppress the $\psi$ in the notation. Then, if $Q=q_{1}$

$$
\gamma(a Q) \gamma^{-1}(t a Q)=\gamma(Q) \gamma^{-1}(t Q)
$$

if and only if $\alpha(a) \alpha(t)=\alpha(a t) \alpha(1)$ if and only if $(a, t)=1$ by (2.1). Since this holds for all $t \in k^{*}$, we conclude this is equivalent to $a \in k^{* 2}$.

For $Q=q_{3}$, the computations imply (2.3) is equivalent to $(a, t)^{3}=1$ for all $t \in k^{*}$. Thus we obtain the same result.

Let $\overline{\mathrm{SL}}_{2}(k)$ be the two-fold covering of $\mathrm{SL}_{2}(k)$. We define a representation $\pi_{Q}^{\psi}$ of $\overline{\mathrm{SL}}_{2}(k)$ on $\mathcal{S}(E)$, the locally constant functions on $E$ with compact support, by specifying it on generators:

$$
\begin{aligned}
\pi_{Q}^{\psi}\left[\left(\begin{array}{cc}
1 & b \\
0 & 1
\end{array}\right), \zeta\right] \Phi(X) & =\zeta^{n} \psi(b Q(X)) \Phi(X), \\
\pi_{Q}^{\psi}\left[\left(\begin{array}{cc}
t & 0 \\
0 & t^{-1}
\end{array}\right), \zeta\right] \Phi(X) & =\zeta^{n}|t|^{n / 2} \gamma_{\psi}(Q) \gamma_{\psi}^{-1}(t Q) \Phi(t X), \\
\pi_{Q}^{\psi}\left[\left(\begin{array}{cc}
0 & 1 \\
-1 & 0
\end{array}\right), \zeta\right] \Phi(X) & =\zeta^{n} \gamma_{\psi}(Q) \hat{\Phi}(X),
\end{aligned}
$$

where $n=\operatorname{dim}_{k} E . \pi_{Q}^{\psi}$ is admissible, dependent on $\psi$, and can be extended to be unitary on $L^{2}(E)$. Note that $\pi_{a Q}^{\psi}$ is the same as $\pi_{Q}^{\psi_{a}}$, and that for the one and three dimensional forms, $\pi_{Q}^{\psi}$ is a genuine (i.e. nontrivial on $\mathbf{Z}_{2}$ ) representation of $\overline{\mathrm{SL}}_{2}(k)$. Thus when we write $Q$, we will, unless otherwise stated, mean a one or a three dimensional anisotropic quadratic form.

Let $\tau$ be a spherical representation of $O(Q)$ acting in $V_{\tau}$. Define $\delta_{\tau}\left(E, V_{\tau}\right)$ to be the space of locally constant maps with compact support from $E$ to $V_{\tau}$ satisfying

$$
f(g X)=\tau(g) f(X), \quad g \in O(Q), X \in E .
$$

If $1_{\tau}$ denotes the trivial representation of $\overline{\mathrm{SL}}_{2}(k)$ on $V_{\tau}$, we define the representation $\pi_{Q, \tau}^{\psi}$ to be the restriction of $\pi_{Q, \tau}^{\psi} \otimes 1_{\tau}$ to the invariant subspace $\mathcal{S}_{\tau}\left(E, V_{\tau}\right)$. (This subspace is invariant because the action of $O(Q)$ commutes with that of $\overline{\mathrm{SL}}_{2}(k)$.) 
We now summarize the basic results proved in [14] about these representations.

THEOREM 2.2. Fix a nontrivial additive character $\psi$.

(a) The representations $\pi_{Q, \tau}^{\psi}$ are irreducible and admissible. Moreover, the map $\tau \mapsto \pi_{Q, \tau}^{\psi}$ is an injection from the set of irreducible spherical representations of $O(Q)$ into the set of irreducible admissible genuine representations of $\overline{\mathrm{SL}}_{2}(k)$.

(b) If $\tau$ is nontrivial, $\pi_{Q, \tau}^{\psi}$ is supercuspidal. If $\tau$ is trivial and $Q=c q_{3}$, then $\pi_{Q, \tau}^{\psi}$ is square-integrable.

(c) If $\pi$ is an irreducible admissible square-integrable genuine representation of $\overline{\mathrm{SL}}_{2}(k)$, then there exists an irreducible representation $\sigma$ of $H^{*} / k^{*}$ and an $a \in k^{*}$ such that $\pi \approx \pi_{a q_{3}, \tilde{\sigma}}^{\psi}$.

(d) If $c \notin k^{* 2}, \pi_{q_{3}, \tilde{x}_{c}}^{\psi} \approx \pi_{-c q_{1}, \theta_{-1}}^{\psi}$.

REMARK 2.3. Theorem 2.2(c) is not exactly the same as that found in [14]; there the representation spaces are Hilbert spaces. However, this poses no difficulty. Call a vector $v$ differentiable if the map $g \mapsto \pi(g) v$ is locally constant. If $(\pi, V)$ is an irreducible admissible square-integrable genuine representation of $\overline{\mathrm{SL}}_{2}(k)$, we can complete $V$ to the Hilbert space $\bar{V}$ and $(\pi, \bar{V})$ will be unitary. Now, by [14], we assert $(\pi, \bar{V}) \approx \pi_{a q_{3}, \tilde{\sigma}}^{\psi}$, the latter acting in the space $L_{\tilde{\sigma}}^{2}\left(H_{0}, V_{\tilde{\sigma}}\right)$ of $L^{2}$ functions from $H_{0}$ to $V_{\tilde{\sigma}}$ satisfying equation (2.7). Moreover, it can be shown that the restriction of $\pi_{a q_{3}, \tilde{\sigma}}^{\psi}$ to the space of differentiable vectors is the irreducible representation $\pi_{a q_{3}, \tilde{\sigma}}^{\psi}$ acting in $\delta_{\tilde{\sigma}}\left(H_{0}, V_{\tilde{\sigma}}\right)$. Since $V \subset \bar{V}_{\text {diff }}$ (the space of differentiable vectors in $\bar{V}$ ), we have $V=\bar{V}_{\text {diff }}$ and Theorem 2.2(c) is obtained.

REMARK 2.4. Theorem 2.2(d) can be generalized to yield all of the equivalences between Weil representations attached to one and three dimensional anisotropic forms. Although we make a conjecture in $\$ 5$, we do not know the possible intertwinings between $\pi_{a q_{3}, \tilde{\sigma}_{1}}^{\psi}$ and $\pi_{b q_{3}, \tilde{\sigma}_{2}}^{\psi}$ for $\sigma_{1}, \sigma_{2}$, representations of $H^{*} / k^{*}$, and $a b^{-1} \notin k^{* 2}$.

We wish to extend the representations $\pi_{Q, \tau}^{\psi}$ to the group $\bar{G}^{*}$, where $\bar{G}^{*}=\{(g, \zeta)$ $\in \bar{G}$ : det $\left.g \in k^{* 2}\right\}$. Let

$$
D=\left\{\left[\left(\begin{array}{cc}
1 & 0 \\
0 & a^{2}
\end{array}\right), 1\right]: a \in k^{*}\right\} .
$$

Then $\bar{G}^{*}$ is the semidirect product of $D$ and $\overline{\operatorname{SL}}_{2}(k)$.

Fix $\pi=\pi_{Q, \tau}^{\psi}$. Let $\lambda \in \hat{k}_{\tau}^{*}($ i.e. $\tau(-I)=\lambda(-1) I)$ and define $\pi$ on $\left(\begin{array}{ll}1 & 0 \\ 0 & a^{2}\end{array}\right)$ by

$$
\pi\left(\begin{array}{cc}
1 & 0 \\
0 & a^{2}
\end{array}\right) \Phi(X)=|a|^{-n / 2} \lambda(a) \Phi\left(a^{-1} X\right) .
$$

Proposition 2.5. For all $\bar{g} \in \overline{\mathrm{SL}}_{2}(k)$,

$$
\pi\left[\left(\begin{array}{cc}
1 & 0 \\
0 & a^{-2}
\end{array}\right) \bar{g}\left(\begin{array}{cc}
1 & 0 \\
0 & a^{2}
\end{array}\right)\right]=\pi\left(\begin{array}{cc}
1 & 0 \\
0 & a^{-2}
\end{array}\right) \pi(\bar{g}) \pi\left(\begin{array}{cc}
1 & 0 \\
0 & a^{2}
\end{array}\right)
$$

that is, $\pi$ extends to an irreducible admissible representation of $\bar{G}^{*}$ which we denote $\pi_{Q, \tau, \lambda}^{\psi}$.

Proof. The above equation is easily verified on the generators $\left.\left[\begin{array}{ll}1 & b \\ 0 & 1\end{array}\right), \zeta\right]$ and $\left[\left(\begin{array}{rr}0 & 1 \\ -1 & 0\end{array}\right), \zeta\right]$. The proof of admissibility proceeds as on pp. 126-128 of [9]. Finally, $\pi_{Q, \tau, \lambda}^{\psi}$ is irreducible since $\pi_{Q, \tau}^{\psi}$ is. 
REMARK 2.6. The center of $\bar{G}^{*}$ is

$$
\bar{Z}=\left\{\left[\left(\begin{array}{cc}
z & 0 \\
0 & z
\end{array}\right), \zeta\right] \in \bar{G}: z \in k^{*}\right\} .
$$

Applying formulas (2.5) and (2.8),

$$
\pi_{Q, \tau, \lambda}^{\psi}(\bar{z})=\omega(\bar{z}) I, \quad \bar{z}=\left[\left(\begin{array}{cc}
z & 0 \\
0 & z
\end{array}\right), \zeta\right] \in \bar{Z},
$$

where $\omega(\bar{z})=\zeta \lambda(z) \gamma_{\psi}(Q) \gamma_{\psi}^{-1}(z Q)$.

Proposition 2.7. $\pi_{a Q, \tau, \lambda}^{\psi} \approx \pi_{Q, \tau, \lambda}^{\psi}$ if and only if $a \in k^{* 2}$.

Proof. $\pi_{a Q, \tau, \lambda}^{\psi} \approx \pi_{Q, \tau, \lambda}^{\psi}$ implies their central characters are equal, and thus $\gamma_{\psi}(Q) \gamma_{\psi}^{-1}(z Q)=\gamma_{\psi}(a Q) \gamma_{\psi}^{-1}(z a Q)$ for all $z \in k^{*}$. By Lemma 2.1, $a \in k^{* 2}$.

For the converse, Proposition 2.27 of [3] and formula (2.8) implies

$$
\pi_{Q, \tau, \lambda}^{\psi}\left[\left(\begin{array}{cc}
1 & 0 \\
0 & a^{-1}
\end{array}\right) \bar{g}\left(\begin{array}{ll}
1 & 0 \\
0 & a
\end{array}\right)\right] \approx \pi_{a Q, \tau, \lambda}^{\psi}(\bar{g}) .
$$

Thus if $a \in k^{* 2}$ the result is clear from Proposition 2.5.

COROLlaRY 2.8. $\pi=\operatorname{Ind}_{\bar{G}^{*} \uparrow \bar{G}} \pi_{q_{3}, \tau, \lambda}^{\psi}$ is irreducible, admissible, and equivalent to $\operatorname{Ind}_{\bar{G}^{*} \uparrow \bar{G}} \pi_{a q_{3}, \tau, \lambda}^{\psi}$ for $a \in \Sigma$. In particular, the induced representation is independent of $\psi$ and we write $\pi=$ Ind $\pi_{q_{3}, \tau, \lambda}$. A similar result holds for $q_{1}$.

Proof. First, note a set of representatives for $\bar{G} / \bar{G}^{*}$ is $\left.\left\{\begin{array}{ll}1 & 0 \\ 0 & a\end{array}\right): a \in \Sigma\right\}$. Formula (2.9) shows how conjugation acts on the representations of $\bar{G}^{*}$ : the representations $\pi_{q_{3}, \tau, \lambda}^{\psi}$ and $\left(\pi_{q_{3}, \tau, \lambda}^{\psi}\right)^{\left(\begin{array}{c}1 \\ 0\end{array}\right)}$ are pairwise inequivalent. Thus, the theory of Mackey (cf. Chapter III, $\$ 13$ of [1]) implies the corollary.

In $\S 3$ it will be desirable to have a model for $\pi_{Q, \tau, \lambda}^{\psi}$ which has a representation space that is easier to work with. We construct this now.

Recall Lemma 1.3 gives a nonzero vector $v_{X}$, invariant by $O(Q)_{X}$, providing that $Q(X) k^{* 2} \in C_{Q, \tau}$. Since $g \in O(Q)$ implies $Q(g X)=Q(X)$, there must be a vector $v_{g X} \neq 0$. We choose the vectors $v_{X}$ such that $\left|v_{X}\right|=\left(v_{X}, v_{X}\right)^{1 / 2}=1$ where, of course, $\tau$ is unitary with respect to this inner product on $V_{\tau}$. Moreover, since $h \in O(Q)_{g X}$ implies $\tau(h) \tau(g) v_{X}=\tau(g) v_{X}$, we can arrange to have $v_{g X}=\tau(g) v_{X}$. Furthermore, since $-I$ is in the center of $O(Q)$, we have $\tau(-I)=\zeta I, \zeta= \pm 1$. Thus

$$
v_{-X}=\zeta v_{X}
$$

We can choose a unitary character $\delta \in \hat{k}_{\tau}^{*}$ and set

$$
v_{t X}=\delta(t) v_{X}, \quad t \in k^{*},
$$

since again $Q(X) k^{* 2} \in C_{Q, \tau}$ implies $Q(t X) k^{* 2} \in C_{Q, \tau}$.

Finally we extend the vectors $v_{X}$ to all $X$ in $E$ as follows: If $\tau \equiv 1$, set $v_{0}=1$. If $\tau \neq 1$, set $v_{0}=0$ and $v_{X}=0$ if $Q(X) k^{* 2} \notin C_{Q, \tau}$.

Consider now a function $\Phi(X) \in \mathcal{S}_{\tau}\left(E, V_{\tau}\right)$. Define a function $\varphi_{\Phi}$ on $Q(E)$ by

$$
\varphi_{\Phi}(Q(X))=|Q(X)|^{n / 4}\left(\Phi(X), v_{X}\right)
$$


A straightforward computation gives:

LEMMA 2.9. $\varphi_{\Phi}$ is well defined and the map $\Phi \mapsto \varphi_{\Phi}$ is a bijection from $\mathcal{\digamma}_{\tau}\left(E, V_{\tau}\right)$ to:

(a) $S\left(k_{Q, \tau}^{*}\right)$ if $\tau \neq 1$ (here $\left.k_{Q, \tau}^{*}=\left\{t \in k^{*}: t k^{* 2} \in C_{Q, \tau}\right\}\right)$, or

(b) functions of the form $|x|^{n / 4} f(x)$ if $\tau \equiv 1$ (here $f(x) \in \mathcal{S}(Q(E))$ ).

Thus we may view $\pi_{Q, \tau, \lambda}^{\psi}$ as acting either in $\delta\left(k_{Q, \tau}^{*}\right)$ or in a space of functions of the form $|x|^{n / 4} f(x)$, with $f \in \mathcal{S}(Q(E))$, according to whether $\sigma$ is nontrivial or trivial. We make the following computations using formulas (2.4)-(2.6), (2.8), (2.10):

$$
\begin{gathered}
\pi_{Q, \tau, \lambda}^{\psi}\left(\begin{array}{cc}
1 & 0 \\
0 & a^{2}
\end{array}\right) \varphi_{\Phi}(Q(X))=\delta^{-1}(a) \lambda(a) \varphi_{\Phi}\left(a^{-2} Q(X)\right), \\
\pi_{Q, \tau, \lambda}^{\psi}\left[\left(\begin{array}{cc}
1 & b \\
0 & 1
\end{array}\right), \zeta\right] \varphi_{\Phi}(Q(X))=\zeta \psi(b Q(X)) \varphi_{\Phi}(Q(X)), \\
\pi_{Q, \tau, \lambda}^{\psi}\left[\left(\begin{array}{cc}
t & 0 \\
0 & t^{-1}
\end{array}\right), \zeta\right] \varphi_{\Phi}(Q(X))=\zeta \delta(t) \gamma_{\psi}(Q) \gamma_{\psi}^{-1}(t Q) \varphi_{\Phi}\left(t^{2} Q(X)\right), \\
\pi_{Q, \tau, \lambda}^{\psi}\left[\left(\begin{array}{cc}
z & 0 \\
0 & z
\end{array}\right), \zeta\right] \varphi_{\Phi}(Q(X))=\zeta \lambda(z) \gamma_{\psi}(Q) \gamma_{\psi}^{-1}(z Q) \varphi_{\Phi}(Q(X)) .
\end{gathered}
$$

REMARK 2.10. Summarizing $\$ 2$, and using the discussion of a matrix of an induced representation found in Chapter III, $\$ 13$, of [1], we can say if $\pi=$ Ind $\pi_{q_{3}, \tilde{\sigma}, \lambda}$, with $\sigma \not \geq 1$ and $\psi$ a fixed nontrivial additive character, then the space of $\pi$ is $\bigoplus_{i=1}^{4} \delta\left(k_{q_{3}, \tilde{\sigma}}^{*}\right)$ and

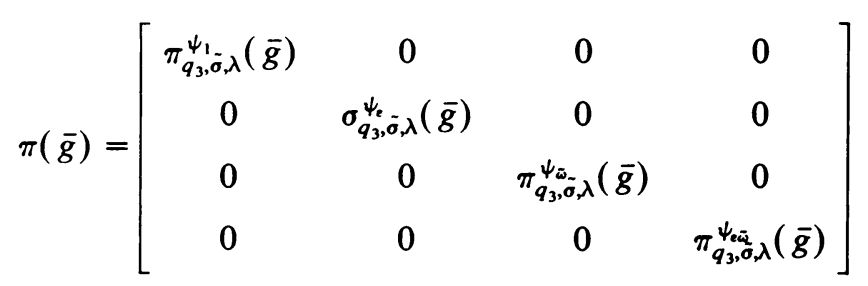

for $\bar{g} \in \bar{G}^{*}$,

$$
\begin{aligned}
& \pi\left(\begin{array}{ll}
1 & 0 \\
0 & \varepsilon
\end{array}\right)=\left[\begin{array}{rrrrrr}
0 & \pi_{q_{3}, \tilde{\sigma}, \lambda}^{\psi_{1}}\left(\begin{array}{cc}
1 & 0 \\
0 & \varepsilon^{2}
\end{array}\right) & 0 & 0 & \\
I & 0 & 0 & 0 & \\
0 & 0 & 0 & \pi_{q_{3}, \tilde{\sigma}, \lambda}^{\psi_{1},}\left(\begin{array}{cc}
1 & 0 \\
0 & \varepsilon^{2}
\end{array}\right) \\
0 & 0 & I & 0 &
\end{array}\right], \\
& \pi\left(\begin{array}{cc}
1 & 0 \\
0 & \tilde{\omega}
\end{array}\right)=\left[\begin{array}{ccrrr}
0 & 0 & \pi_{q_{3}, \tilde{\sigma}, \lambda}^{\psi_{1},}\left(\begin{array}{cc}
1 & 0 \\
0 & \tilde{\omega}^{2}
\end{array}\right) & 0 & \\
0 & 0 & 0 & \pi_{q_{3}, \tilde{\sigma}, \lambda}^{\psi_{1}, \lambda}\left(\begin{array}{cc}
1 & 0 \\
0 & \tilde{\omega}^{2}
\end{array}\right) \\
I & 0 & 0 & 0 \\
0 & I & 0 & 0
\end{array}\right],
\end{aligned}
$$


and

$$
\pi\left(\begin{array}{cc}
1 & 0 \\
0 & \varepsilon \tilde{\omega}
\end{array}\right)=\left[\begin{array}{rrrr}
0 & 0 & 0 & \pi_{q_{3}, \tilde{\sigma}, \lambda}^{\psi_{1}}\left(\begin{array}{cc}
1 & 0 \\
0 & \varepsilon^{2} \tilde{\omega}^{2}
\end{array}\right) \\
0 & 0 & \pi_{q_{3}, \tilde{\sigma}, \lambda}^{\psi_{1}}\left(\begin{array}{cc}
1 & 0 \\
0 & \tilde{\omega}^{2}
\end{array}\right) & 0 \\
0 & \pi_{q_{3}, \tilde{\sigma}, \lambda}^{\psi_{1}}\left(\begin{array}{cc}
1 & 0 \\
0 & \varepsilon^{2}
\end{array}\right) & 0 & 0 \\
1 & 0 & 0 & 0
\end{array}\right]
$$

where

$$
\begin{aligned}
& \pi_{q_{3}, \tilde{\sigma}, \lambda}^{\psi_{\alpha}}\left[\left(\begin{array}{ll}
1 & b \\
0 & 1
\end{array}\right), \zeta\right] \varphi(x)=\zeta \psi(\alpha b x) \varphi(x), \\
& \pi_{q_{3}, \tilde{\sigma}, \lambda}^{\psi_{\alpha}, \lambda}\left[\left(\begin{array}{ll}
z & 0 \\
0 & z
\end{array}\right), \zeta\right] \varphi(x)=\zeta \lambda(z) \gamma_{\psi}\left(\alpha q_{3}\right) \gamma_{\psi}^{-1}\left(z \alpha q_{3}\right) \varphi(x),
\end{aligned}
$$

for $x \in k_{q_{3}, \tilde{\sigma}}^{*}$ and $\alpha \in \Sigma$.

For $\sigma \equiv 1$ the formulas are the same, but the representation space is $\bigoplus_{i=1}^{4}$ space of functions of the form $|x|^{3 / 4} f(x)$ for $f(x) \in \mathcal{S}\left(q_{3}\left(H_{0}\right)\right)$ (Lemma 2.9).

Finally, analogous formulas hold for the case $Q=q_{1}$.

3. Whittaker functionals-calculation of $\Omega_{\psi}(\pi)$. Before stating the main theorem of this section, we need to review briefly $\$ 3$ of [6].

Let $p: \bar{G} \rightarrow G$ denote the projection $(g, \zeta) \mapsto g$. If $H$ is any subgroup of $G$, denote $p^{-1}(H)$ by $\bar{H}$.

Set

$$
Z^{n}=\left\{\left(\begin{array}{cc}
z^{n} & 0 \\
0 & z^{n}
\end{array}\right): z \in k^{*}\right\} \simeq k^{* n}
$$

Then $\overline{Z^{2}} \simeq Z^{2} \times \mathbf{Z}_{2}$ is the center of $\bar{G}$.

If $(\pi, V)$ is an irreducible admissible representation of $\bar{G}$, then there is a quasi-character $\omega_{\pi}$ of $\overline{Z^{2}}$ such that $\pi(\bar{z})=\omega_{\pi}(\bar{z}) I, \bar{z} \in \overline{Z^{2}}$.

Let $\psi$ be a nontrivial additive character. Define $\Omega\left(\omega_{\pi}\right)$ to be the (finite) set of quasi-characters of $\bar{Z}=\overline{Z^{1}}$ that agree with $\omega_{\pi}$ on $\overline{Z^{2}}$, and define $\Omega_{\psi}(\pi)$ to be those $\mu \in \Omega\left(\omega_{\pi}\right)$ such that there exists a nonzero linear functional $l$ on $V$ satisfying

$$
l\left(\pi\left(\begin{array}{ll}
1 & b \\
0 & 1
\end{array}\right) v\right)=\psi(b) l(v), \quad b \in k, v \in V
$$

and $l(\pi(\bar{z}) v)=\mu(\bar{z}) l(v), z \in \bar{Z}, v \in V$. Such an $l$ is unique up to a constant (Theorem 4.1 of [7]) and is called a $(\psi, \mu)$ Whittaker functional. We remark that $\# \Omega_{\psi}(\pi)$ is independent of $\psi$, and $\# \Omega_{\psi}(\pi)>0$ if $\pi$ is infinite dimensional. We say a representation $\pi$ of $\bar{G}$ is distinguished if $\# \Omega_{\psi}(\pi)=1$ for some (hence all) $\psi$.

THEOREM 3.1. Suppose $\psi$ is a nontrivial additive character of $k$.

(1) Let $\pi=$ Ind $\pi_{q_{3}, \tilde{\sigma}, \lambda}, \lambda \in \widehat{k_{\tilde{\sigma}}^{*}}$.

(a) If $\sigma \equiv 1, \Omega_{\psi}(\pi)=\left\{\mu_{1}, \mu_{2}, \mu_{3}\right\}$, where

$$
\mu_{i}(z, \zeta)=\zeta \lambda(z) \gamma_{\psi}\left(a_{i} q_{3}\right) \gamma_{\psi}^{-1}\left(z a_{i} q_{3}\right), \quad i=1,2,3,
$$

and $a_{1}, a_{2}, a_{3}$ are the three $k^{* 2}$ coset representatives in $q_{3}\left(H_{0}^{*}\right)$. 
(b) If $\sigma=\chi_{b}, b \notin k^{* 2}, \Omega_{\psi}(\pi)=\{\mu\}$, where

$$
\mu(z, \zeta)=\zeta \lambda(z) \gamma_{\psi}\left(-b q_{3}\right) \gamma_{\psi}^{-1}\left(-z b q_{3}\right) \text {. }
$$

(c) If $\operatorname{dim} \sigma>1, \Omega_{\psi}(\pi)=\left\{\mu_{1}, \mu_{2}\right\}$, where

$$
\mu_{i}(z, \zeta)=\zeta \lambda(z) \gamma_{\psi}\left(e_{i} q_{3}\right) \gamma_{\psi}^{-1}\left(z e_{i} q_{3}\right), \quad i=1,2,
$$

and $e_{1}, e_{2}$ are two distinct $k^{* 2}$ coset representatives in $q_{3}\left(H_{0}^{*}\right)$ such that $\left(\alpha(\sigma),-e_{1}\right)=$ $\left(\alpha(\sigma),-e_{2}\right)=-1$.

(2) Let $\pi=$ Ind $\pi_{q_{1}, \theta, \lambda}$ with $\theta=\theta_{1}$ or $\theta_{-1}$ and $\lambda \in \widehat{k_{\theta}^{*}}$. Then $\Omega_{\psi}(\pi)=\{\mu\}$, where

$$
\mu(z, \zeta)=\zeta \lambda(z) \gamma_{\psi}\left(q_{1}\right) \gamma_{\psi}^{-1}\left(z q_{1}\right) \text {. }
$$

Proof. We first prove the cases (1b), (1c), and (2), $\theta=\theta_{-1}$, i.e. for the nontrivial representations of the orthogonal groups. By Lemma 2.9 and Theorem 1.6, the space of $\pi_{q_{3}, \tilde{x} b, \lambda}^{\psi}, b \notin k^{* 2}$ is $\delta\left(-b k^{* 2}\right)$. In particular there is a $\varphi \in \delta\left(-b k^{* 2}\right)$ such that $\varphi\left(\alpha^{-1}\right) \neq 0$, where $\alpha \in \Sigma$ is congruent to $-b$ modulo $k^{* 2}$. According to Remark 2.10, we may view $\left.\pi\right|_{G^{*}}$ as the matrix (2.11) acting on

$$
\left[\begin{array}{c}
\varphi_{1} \\
\varphi_{\varepsilon} \\
\varphi_{\omega} \\
\varphi_{e \tilde{\omega}}
\end{array}\right], \quad \varphi_{\beta} \in \mathcal{S}\left(-b k^{* 2}\right), \beta \in \Sigma .
$$

Define a linear functional $L$ by

$$
L\left[\begin{array}{c}
\varphi_{1} \\
\varphi_{e} \\
\varphi_{\tilde{\omega}} \\
\varphi_{e \tilde{\omega}}
\end{array}\right]=\varphi_{\alpha}\left(\alpha^{-1}\right)
$$

$L$ is nonzero and

$$
\begin{aligned}
L\left[\left(\begin{array}{ll}
1 & b \\
0 & 1
\end{array}\right)\left[\begin{array}{c}
\varphi_{1} \\
\vdots \\
\varphi_{e \tilde{\omega}}
\end{array}\right]\right) & =L\left[\begin{array}{c}
\psi_{1}(b x) \varphi_{1}(x) \\
\vdots \\
\psi_{e \tilde{\omega}}(b x) \varphi_{e \tilde{\omega}}(x)
\end{array}\right]=\left.\psi_{\alpha}(b x) \varphi_{\alpha}(x)\right|_{x=\alpha^{-1}} \\
& =\psi(b) \varphi_{\alpha}\left(\alpha^{-1}\right)=\psi(b) L\left[\begin{array}{c}
\varphi_{1} \\
\vdots \\
\varphi_{e \tilde{\omega}}
\end{array}\right] \\
L\left[\pi(\bar{z})\left[\begin{array}{c}
\varphi_{1} \\
\vdots \\
\varphi_{\varepsilon \tilde{\omega}}
\end{array}\right]\right) & =L\left[\begin{array}{c}
\zeta \lambda(z) \gamma_{\psi}\left(q_{3}\right) \gamma_{\psi}^{-1}\left(z q_{3}\right) \varphi_{1}(x) \\
\vdots \\
\zeta \lambda(z) \gamma_{\psi}\left(\varepsilon \tilde{\omega} q_{3}\right) \gamma_{\psi}^{-1}\left(z \varepsilon \tilde{\omega} q_{3}\right) \varphi_{e \tilde{\omega}}(x)
\end{array}\right] \\
& =\left.\zeta \lambda(z) \gamma_{\psi}\left(\alpha q_{3}\right) \gamma_{\psi}^{-1}\left(z \alpha q_{3}\right) \varphi_{\alpha}(x)\right|_{x=\alpha^{-1}} \\
& =\mu(z, \zeta) L\left[\begin{array}{c}
\varphi_{1} \\
\vdots \\
\varphi_{\tilde{L} \tilde{\omega}}
\end{array}\right]
\end{aligned}
$$


where $\mu(z, \zeta)=\zeta \lambda(z) \gamma_{\psi}\left(\alpha q_{3}\right) \gamma_{\psi}^{-1}\left(z \alpha q_{3}\right)=\zeta \lambda(z) \gamma_{\psi}\left(-b q_{3}\right) \gamma_{\psi}^{-1}\left(-z b q_{3}\right)$. That is, $L$ is a $(\psi, \mu)$ Whittaker functional.

For case (1c), Lemma 2.9 and Theorem 1.6 show $S\left(\alpha_{1} k^{*} \cup \alpha_{2} k^{* 2}\right)$ is the space of $\pi_{q_{3}, \tilde{\sigma}, \lambda}^{\psi}$ where $\alpha_{i} \in \Sigma$ are such that $\left(\alpha(\sigma),-\alpha_{i}\right)=-1$. Define two linear functionals $L_{1}, L_{2}$ by

$$
L_{i}\left[\begin{array}{c}
\varphi_{1} \\
\vdots \\
\varphi_{\varepsilon \tilde{\omega}}
\end{array}\right]=\varphi_{\alpha_{i}}\left(\alpha_{i}^{-1}\right), \quad i=1,2
$$

As above, we verify the $L_{i}$ are $\left(\psi, \mu_{i}\right)$ Whittaker functionals with $\mu_{i}(z, \zeta)=$ $\zeta \lambda(z) \gamma_{\psi}\left(\alpha_{i} q_{3}\right) \gamma_{\psi}^{-1}\left(z \alpha_{i} q_{3}\right), i=1,2$.

For $Q=q_{1}$, we mimic case (lb) and omit the details.

Having constructed these $(\psi, \mu)$ Whittaker functionals we must show they are the only possible ones (up to a scalar multiple). Note that if $L$ is a $(\psi, \mu)$ functional on $V$, then

$$
L\left[\begin{array}{c}
\varphi_{1} \\
\varphi_{\varepsilon} \\
\varphi_{\tilde{\omega}} \\
\varphi_{\varepsilon \tilde{\omega}}
\end{array}\right]=L\left[\begin{array}{c}
\varphi_{1} \\
0 \\
0 \\
0
\end{array}\right]+L\left[\begin{array}{c}
0 \\
\varphi_{e} \\
0 \\
0
\end{array}\right]+L\left[\begin{array}{c}
0 \\
0 \\
\varphi_{\tilde{\omega}} \\
0
\end{array}\right]+L\left[\begin{array}{c}
0 \\
0 \\
0 \\
\varphi_{\varepsilon \tilde{\omega}}
\end{array}\right]
$$

Claim $L$ is nonzero only on one of the subspaces

$$
\left[\begin{array}{c}
0 \\
\dot{\cdot} \\
\varphi_{\alpha} \\
\dot{0}
\end{array}\right], \quad \alpha \in \Sigma .
$$

Indeed, say

$$
L\left[\begin{array}{c}
\varphi_{1} \\
0 \\
0 \\
0
\end{array}\right] \neq 0 \neq L\left[\begin{array}{c}
0 \\
\varphi_{e} \\
0 \\
0
\end{array}\right]
$$

Then

$$
\begin{aligned}
& \mu(\bar{z}) L\left[\begin{array}{c}
\varphi_{1} \\
0 \\
0 \\
0
\end{array}\right]=L\left[\pi(\bar{z})\left[\begin{array}{c}
\varphi_{1} \\
0 \\
0 \\
0
\end{array}\right]\right)=\zeta \lambda(z) \gamma_{\psi}(Q) \gamma_{\psi}^{-1}(z Q) L\left[\begin{array}{c}
\varphi_{1} \\
0 \\
0 \\
0
\end{array}\right], \\
& \mu(\bar{z}) L\left[\begin{array}{c}
0 \\
\varphi_{\varepsilon} \\
0 \\
0
\end{array}\right]=L\left(\pi(\bar{z})\left[\begin{array}{c}
0 \\
\varphi_{\varepsilon} \\
0 \\
0
\end{array}\right]\right)=\zeta \lambda(z) \gamma_{\psi}(\varepsilon Q) \gamma_{\psi}^{-1}(z \varepsilon Q) L\left[\begin{array}{c}
0 \\
\varphi_{\varepsilon} \\
0 \\
0
\end{array}\right],
\end{aligned}
$$

and thus $\gamma_{\psi}(Q) \gamma_{\psi}^{-1}(z Q)=\gamma_{\psi}(\varepsilon Q)=\gamma_{\psi}^{-1}(z \varepsilon Q)$ contradicting Lemma 2.1. The proof of cases (lb), (lc), and (2), $\theta=\theta_{-1}$ is completed by applying the following lemma. 
LEMMA 3.2. Suppose $T$ is a linear functional on the space of functions $\mathcal{S}(\mathbb{Q})$, where $\mathbb{Q}$ is a fixed open set in $k^{*}$. Suppose also that for a fixed $r \in k^{*}$,

$$
T\left(\psi_{r}(b x) \varphi(x)\right)=\psi(b) T(\varphi(x)), \quad b \in k,
$$

for all $\varphi \in S(\mathbb{Q})$. Then, up to a scalar multiple, $T(\varphi(x))=\varphi\left(r^{-1}\right)$.

Proof. See p. 59 of [9].

The remaining two cases in Theorem 3.1 are the cases Ind $\pi_{q_{3}, \tilde{\sigma}, \lambda}$ with $\sigma \equiv 1$ and Ind $\pi_{q_{1}, \theta_{1}, \lambda}$. The construction of the $(\psi, \mu)$ Whittaker functionals is exactly the same as before. All we must show is the analogue of Lemma 3.2 for a space of functions of the form $|x|^{n / 4} f(x), f(x) \in \mathcal{S}(Q(E))$. We use the following technique to reduce the proof to an application of Lemma 3.2.

Take $\varphi$ of the form $|x|^{n / 4} f(x)$ and choose an $n \in k$ such that $\psi(n) \neq 1$. Note that $\varphi(x)-\psi(r n x) \varphi(x)$ is in $\mathcal{S}\left(Q\left(E^{*}\right)\right)$ and Lemma 3.2 applies to give

$$
\begin{aligned}
T(\varphi(x)) & =T(\varphi(x)-\psi(r n x) \varphi(x))+T(\psi(r n x) \varphi(x)) \\
& =c\left(\varphi\left(r^{-1}\right)-\psi\left(r n r^{-1}\right) \varphi\left(r^{-1}\right)\right)+\psi(n) T(\varphi(x)) .
\end{aligned}
$$

That is, $(1-\psi(n)) T(\varphi(x))=c(1-\psi(n)) \varphi\left(r^{-1}\right)$. Thus $T(\varphi(x))=c \varphi\left(r^{-1}\right)$ as desired.

With this the proof of Theorem 3.1 is complete.

Recall a representation $\pi$ of $\bar{G}$ is genuine if it is nontrivial on $\mathbf{Z}_{2}$. Certainly all nongenuine $\pi$, i.e. ordinary representations of $\mathrm{GL}_{2}(k)$, are distinguished since $\pi$ operates as a scalar on $\bar{Z}$. Before determining the genuine distinguished representations of $\bar{G}$, we need a few equivalences.

First, recall the classification of the irreducible admissible nonsupercuspidal representations of $\bar{G}$ (cf. [13] and [11]).

Let $B_{2}$ be the subgroup of matrices of the form

$$
\left(\begin{array}{cc}
a_{1} & x \\
0 & a_{2}
\end{array}\right)
$$

where the $a_{i}$ have even order. Then define, for any quasi-characters $\mu_{1}$ and $\mu_{2}$ of $k^{*}$,

$$
\mu_{1} \mu_{2}\left[\left(\begin{array}{cc}
a_{1} & x \\
0 & a_{2}
\end{array}\right), \zeta\right]=\zeta \mu_{1}\left(a_{1}\right) \mu_{2}\left(a_{2}\right) \text {. }
$$

Let $\bar{\rho}\left(\mu_{1}, \mu_{2}\right)=\operatorname{Ind}_{B_{2} \uparrow \bar{G}} \mu_{1} \mu_{2}$. We remark that multiplying the $\mu_{i}$ by characters of order 2 is irrelevant. We have similarly to $\mathrm{GL}_{2}(k)$ :

(a) If $\left(\mu_{1} \mu_{2}^{-1}\right)^{2} \neq||$ or ||$^{-1}$, then $\bar{\rho}\left(\mu_{1}, \mu_{2}\right)$ is irreducible and denoted by $\bar{\pi}\left(\mu_{1}, \mu_{2}\right)$;

(b) if $\left(\mu_{1} \mu_{2}^{-1}\right)^{2}=||^{-1}, \bar{\rho}\left(\mu_{1}, \mu_{2}\right)$ contains one subrepresentation denoted $\bar{\pi}\left(\mu_{1}, \mu_{2}\right)$;

(c) if $\left(\mu_{1} \mu_{2}^{-1}\right)^{2}=||, \bar{\rho}\left(\mu_{1}, \mu_{2}\right)$ contains one subrepresentation (a "special" representation) denoted $\bar{\pi}\left(\mu_{1}, \mu_{2}\right)$.

Proposition 3.3. (a) Ind $\pi_{q_{3}, \tilde{x}_{b}, x_{b} \lambda} \approx$ Ind $\pi_{q_{1}, \theta_{-1}, \lambda}$ for any $b \notin k^{* 2}$ and $\lambda$ with $\lambda(-1)=-1$

(b) Ind $\pi_{q_{1}, \theta_{1}, \lambda} \approx \bar{\pi}\left(\lambda^{1 / 2}||^{-1 / 4}, \lambda^{1 / 2}||^{1 / 4}\right), \lambda(-1)=1$;

(c) Ind $\pi_{q_{3}, \tilde{\sigma}, \lambda} \approx \bar{\pi}\left(\lambda^{1 / 2}||^{1 / 4}, \lambda^{1 / 2}||^{-1 / 4}\right)$, if $\sigma \equiv 1, \lambda(-1)=1$. 
Proof. (a) It suffices to show $\pi_{q_{3}, \tilde{x}_{b}, x_{b} \lambda} \approx \pi_{-b q_{1}, \theta_{-1}, \lambda}$ for $b \notin k^{* 2}$ and fixed $\psi$. Note $\chi_{b} \lambda \in \widehat{k_{\chi_{b}}^{*}}$ so the left-hand side makes sense. Indeed by Corollary 1.9, $\tilde{\chi}_{b}(-I)=$ $-(b,-1) I=\chi_{b} \lambda(-1) I$. Theorem $2.2(\mathrm{~d})$ gives $\pi_{q_{3}, \tilde{\chi}_{b}}^{\psi} \approx \pi_{-b q_{1}, \theta_{-1}}$ and so by Schur's lemma

$$
\pi_{q_{3}, \bar{x}_{b}, x_{b} \lambda}^{\psi} \approx \pi_{-b q_{1}, \theta_{-1}, x}^{\psi}
$$

for some $\chi \in \widehat{k_{\theta_{-1}^{*}}^{*}}$. Computing central characters gives $\zeta \chi_{b} \lambda(z) \gamma_{\psi}\left(q_{3}\right) \gamma_{\psi}^{-1}\left(z q_{3}\right)=$ $\zeta \chi(z) \gamma_{\psi}\left(-b q_{1}\right) \gamma_{\psi}^{-1}\left(-b z q_{1}\right)$ i.e.

$$
\begin{gathered}
\frac{\chi_{b} \lambda(z) \alpha_{\psi}(-\varepsilon) \alpha_{\psi}(-\tilde{\omega}) \alpha_{\psi}(\varepsilon \tilde{\omega})}{\alpha_{\psi}(-\varepsilon z) \alpha_{\psi}(-\tilde{\omega} z) \alpha_{\psi}(\varepsilon \tilde{\omega} z)}=\frac{\chi(z) \alpha_{\psi}(-b)}{\alpha_{\psi}(-b z)} \\
\chi_{b} \lambda(z)(-\varepsilon, z)(-\tilde{\omega}, z)(\varepsilon \tilde{\omega}, z)\left[\frac{\alpha_{\psi}(1)}{\alpha_{\psi}(z)}\right]^{3}=\chi(z)(-b, z) \frac{\alpha_{\psi}(1)}{\alpha_{\psi}(z)} .
\end{gathered}
$$

So

$$
\chi_{b} \lambda(z)\left[\frac{\alpha_{\psi}(1)}{\alpha_{\psi}(z)}\right]^{2}=(-b, z) \chi(z) .
$$

But $\alpha_{\psi}(z)^{2}=\alpha_{\psi}(z) \alpha_{\psi}(z)=\alpha_{\psi}\left(z^{2}\right) \alpha_{\psi}(1)(z, z)=\alpha_{\psi}(1)^{2}(-1, z)$. So we obtain

$$
\chi_{b} \lambda(z)(-1, z)=(-b, z) \chi(z)
$$

and so $\chi(\mathrm{z})=\lambda(\mathrm{z})$ proving $(\mathrm{a})$.

(b) This is Proposition 2.3.3 of [5].

(c) Certainly Ind $\pi_{q_{3}, \tilde{\sigma}, \lambda}$ must be a $\bar{\pi}\left(\chi||^{1 / 4},\left.\chi\right|^{-1 / 4}\right)$ for some $\chi$. Computing central characters gives $\lambda\left(z^{2}\right)=\chi^{2}\left(z^{2}\right)$, i.e. $\lambda^{1 / 2}=\chi$ on squares. This suffices since multiplication by characters of order 2 is irrelevant in describing the principal series.

Suppose $\lambda$ is a quasi-character of $k^{*}$. Define

$$
R_{\lambda}= \begin{cases}\text { Ind } \pi_{q_{1}, \theta_{1}, \lambda} & \text { if } \lambda(-1)=1, \\ \text { Ind } \pi_{q_{1}, \theta_{-}, \lambda} & \text { if } \lambda(-1)=-1\end{cases}
$$

For the moment we shall assume a result of the next section:

THEOREM 3.4. Every irreducible admissible genuine supercuspidal representation of $\bar{G}$ is an Ind $\pi_{q_{3}, \tilde{\sigma}, \lambda}$, for some $\sigma \neq 1$ and $\lambda \in \widehat{k_{\tilde{\sigma}}^{*}}$.

Using 3.4 we can prove Conjecture 4.2 of [4]:

THEOREM 3.5. The map $\lambda \mapsto R_{\lambda}$ is a bijection between the set of quasi-characters of $k^{*}$ and the set of irreducible admissible genuine distinguished representations of $\bar{G}$.

Proof. Among the supercuspidal representations, Theorems 3.4, 3.1(1b), (1c) and Proposition 3.3(a) show that only the $R_{\lambda}$ with $\lambda(-1)=-1$ are distinguished. Among the principal series, for $\bar{\pi}=\pi\left(\mu_{1}, \mu_{2}\right)$, with $\left(\mu_{1} \mu_{2}^{-1}\right)^{2}(x) \neq|x|$ or $|x|^{-1}$, Theorem 2.4 of [5] shows that $\Omega_{\psi}(\bar{\pi})=\Omega\left(\omega_{\bar{\pi}}\right)$ so that $\# \Omega_{\psi}(\bar{\pi})=4$. In the remaining two cases when $\left(\mu_{1} \mu_{2}^{-1}\right)^{2}(x)=|x|$ or $|x|^{-1}$, Theorem 3.1(1a), (2) and Proposition 3.3(b), 3.3(c) show that only the $R_{\lambda}$ with $\lambda(-1)=1$ are distinguished. Thus $\lambda \mapsto R_{\lambda}$ is surjective. 
To show the map is injective, suppose $R_{\lambda_{1}} \approx R_{\lambda_{2}}$. Clearly $\lambda_{1}(-1)=\lambda_{2}(-1)$, otherwise one representation would be supercuspidal and the other would not. So we have Ind $\pi_{q_{1}, \theta_{m}, \lambda_{1}} \approx$ Ind $\pi_{q_{1}, \theta_{m}, \lambda_{2}}, m=\lambda_{1}(-1)=\lambda_{2}(-1)$, that is,

$$
\pi_{q_{1}, \theta_{m}, \lambda_{1}}^{\psi} \approx\left(\pi_{q_{1}, \theta_{m}, \lambda_{2}}^{\psi}\right)^{\left(\begin{array}{ll}
1 & 0 \\
0
\end{array}\right)} \approx \pi_{b q_{1}, \theta_{m}, \lambda_{2}}^{\psi}
$$

for some $b \in \Sigma$. Restricting to $\overline{\mathrm{SL}}_{2}(k)$ gives $\pi_{q_{1}, \theta_{m}}^{\psi} \approx \pi_{b q_{1}, \theta_{m}}^{\psi}$ and, since it is shown in [14] that $\pi_{Q_{1}, \tau_{1}}^{\psi} \approx \pi_{Q_{2}, \tau_{2}}^{\psi}$ implies $C_{Q_{1}, \tau_{1}}=C_{Q_{2}, \tau_{2}}$, we have

$$
k^{* 2}=C_{q_{1}, \theta_{m}}=C_{b q_{1}, \theta_{m}}=b k^{* 2} \text {. }
$$

Thus $b \in k^{* 2}$ and, comparing the central characters of the representations on both sides of (3.2), we obtain $\lambda_{1}=\lambda_{2}$.

4. Construction of supercuspidal representations of $\bar{G}$. We turn now to showing that any irreducible admissible genuine supercuspidal representation of $\bar{G}$ is an Ind $\pi_{q_{3}, \tilde{\sigma}, \lambda}$ for some nontrivial irreducible representation $\sigma$ of $H^{*} / k^{*}$.

First, suppose that we could obtain all the irreducible admissible unitary genuine supercuspidals as Ind $\pi_{q_{3}, \tilde{\sigma}, \lambda}$ with $\sigma$ nontrivial and $\lambda$ unitary. If we knew that given an arbitrary genuine supercuspidal, there is a quasi-character $\chi$ such that $\chi \otimes \pi$ is unitary, then

$$
\chi \otimes \pi=\text { Ind } \pi_{q_{3}, \tilde{\sigma}, \lambda}
$$

implying

$$
\pi=\text { Ind } \pi_{q_{3}, \tilde{\sigma}, \lambda x^{2}}
$$

and we would be done. That such a $\chi$ exists is a consequence of the following proposition.

Proposition 4.1. Let $\pi$ be an irreducible admissible supercuspidal representation of $\bar{G}$ with

$$
\pi(\bar{z})=\omega_{\pi}(\bar{z}) I, \quad \bar{z} \in \overline{Z^{2}} .
$$

If $\omega_{\pi}$ is unitary, then so is $\pi$.

Proof. Consider the Kirillov model for $\pi$. The representation space is $V=$ $\bigoplus_{\mu \in \Omega_{\downarrow}(\pi)} \delta\left(k^{*}\right)$ where $\pi$ acts through the formulas

$$
\begin{gathered}
\pi\left(\begin{array}{ll}
a & b \\
0 & 1
\end{array}\right) \varphi^{\mu}(x)=\psi(b x) \varphi^{\mu}(a x), \quad a \in k^{*}, b \in k, \\
\pi(\bar{z}) \varphi^{\mu}(x)=(x, z) \mu(\bar{z}) \varphi^{\mu}(x), \quad \bar{z} \in \bar{Z} .
\end{gathered}
$$

For details, see $\$ 3$ of [5]. We have to construct a positive definite hermitian invariant form on $V \times V$.

For $f$ and $g$ in $\delta\left(k^{*}\right)$ set

$$
\langle f, g\rangle_{1}=\int_{k^{*}} f(x) g(x) d^{*} x
$$

and on $V$, set

$$
\langle\Phi, \Psi\rangle=\left\langle\left(\Phi_{1}, \ldots, \Phi_{n}\right),\left(\Psi_{1}, \ldots, \Psi_{n}\right)\right\rangle=\sum_{i=1}^{n}\left\langle\Phi_{i}, \Psi_{i}\right\rangle_{1} .
$$

Here $n=\# \Omega(\pi)$ and we have fixed an order on the $\mu \in \Omega(\pi)$. 
For $\Phi$ and $\tilde{\Phi}$ in $V$ consider the function $f_{\Phi, \tilde{\Phi}}(g)=\langle\pi(g) \Phi, \tilde{\Phi}\rangle$. Claim there is a compact set $C=C_{\Phi, \tilde{\Phi}}$ such that $\operatorname{supp} f_{\Phi, \tilde{\Phi}} \subset \bar{Z} C$. We have $\bar{G}=K \bar{A} K$ with $K=$ $\mathrm{GL}_{2}\left(O_{k}\right)$ and

$$
\bar{A}=\left\{\left[\left(\begin{array}{ll}
a & 0 \\
0 & b
\end{array}\right), \zeta\right]: a, b \in k^{*}\right\}
$$

Since $\Phi$ and $\tilde{\Phi}$ are both invariant under subgroups of finite index in $K$, we need only show $f_{\boldsymbol{\Phi}, \tilde{\Phi}}$ on $\bar{A}$ has its support contained in $\bar{Z} C$. Since

$$
\begin{aligned}
\left\langle\pi\left(\left[\left(\begin{array}{ll}
a & 0 \\
0 & a
\end{array}\right), \zeta\right]\left(\begin{array}{ll}
b & 0 \\
0 & 1
\end{array}\right)\right) \Phi, \tilde{\Phi}\right\rangle & =\sum_{i=1}^{n}\left\langle\pi\left[\left(\begin{array}{ll}
a & 0 \\
0 & a
\end{array}\right), \zeta\right] \pi\left(\begin{array}{ll}
b & 0 \\
0 & 1
\end{array}\right) \Phi_{i}, \tilde{\Phi}_{i}\right\rangle_{1} \\
& =\sum_{i=1}^{n} \mu_{i}\left(\left[\left(\begin{array}{ll}
a & 0 \\
0 & a
\end{array}\right), \zeta\right]\right)\left\langle(x, a) \pi\left(\begin{array}{ll}
b & 0 \\
0 & 1
\end{array}\right) \Phi_{i}(x), \tilde{\Phi}_{i}(x)\right\rangle_{1}
\end{aligned}
$$

we need only show the function of $b$ given by

$$
\left\langle(x, a) \pi\left(\begin{array}{ll}
b & 0 \\
0 & 1
\end{array}\right) \Phi_{1}(x), \tilde{\Phi}_{1}(x)\right\rangle_{1}
$$

has compact support in $k^{*}$ (independent of $a$ ). For then so would $\left\langle(x, a) \pi\left(\begin{array}{ll}b & 0 \\ 0 & 1\end{array}\right) \Phi_{i}(x), \tilde{\Phi}_{i}(x)\right\rangle_{1}, i=2, \ldots, n$, and the support of the sum is contained in the union of the supports. But

$$
\left\langle(x, a) \pi\left(\begin{array}{ll}
b & 0 \\
0 & 1
\end{array}\right) \Phi_{1}(x), \tilde{\Phi}_{1}(x)\right\rangle_{1}=\int_{k^{*}}(x, a) \Phi_{1}(b x) \tilde{\Phi}_{1}(x) d^{*} x,
$$

and since $\Phi_{1}$ and $\tilde{\Phi}_{1}$ have compact supports in $k^{*}$, the result is clear.

Thus supp $f_{\Phi, \tilde{\Phi}} \subset \bar{Z} C$ implying supp $f_{\Phi, \tilde{\Phi}} \subset \overline{Z^{2}} C^{\prime}$ since $\left[\overline{Z:} \overline{Z^{2}}\right]<\infty$.

Choose now a fixed nonzero function $\Phi^{0}$ in $V$ and define on $V \times V$

$$
(\Phi, \Psi)=\int_{\overline{Z^{2}} / \bar{G}}\left\langle\pi(g) \Phi, \Phi^{0}\right\rangle\left\langle\overline{\pi(g) \Psi, \Phi^{0}}\right\rangle d g .
$$

Note the integrand is indeed $\overline{Z^{2}}$ invariant;

$$
\begin{aligned}
\left\langle\pi\left(\bar{z}^{2}\right) \Phi, \Phi^{0}\right\rangle\left\langle\overline{\pi\left(\bar{z}^{2}\right) \Psi, \Phi^{0}}\right\rangle & =\left[\sum_{i=1}^{n}\left\langle\omega_{\pi}\left(\bar{z}^{2}\right) \Phi_{i}, \Phi_{i}^{0}\right\rangle_{1}\right]\left[\overline{\sum_{i=1}^{n}\left\langle\omega_{\pi}\left(\bar{z}^{2}\right) \Psi_{i}, \Phi_{i}^{0}\right\rangle_{1}}\right] \\
& =\omega_{\pi}\left(\bar{z}^{2}\right)\left[\sum_{i=1}^{n}\left\langle\Phi_{i}, \Phi_{i}^{0}\right\rangle_{1}\right] \bar{\omega}_{\pi}\left(\bar{z}^{2}\right)\left[\overline{\sum_{i=1}^{n}\left\langle\Psi_{i}, \Phi_{i}^{0}\right\rangle_{1}}\right] \\
& =\left[\sum_{i=1}^{n}\left\langle\Phi_{i}, \Phi_{i}^{0}\right\rangle_{1}\right] \overline{\left[\sum_{i=1}^{n}\left\langle\Psi_{i}, \Phi_{i}^{0}\right\rangle_{1}\right]} \text { since } \omega_{\pi} \text { is unitary } \\
& =\left\langle\Phi, \Phi^{0}\right\rangle\left\langle\overline{\Psi, \Psi^{0}}\right\rangle .
\end{aligned}
$$

One can easily check that $(\Phi, \Psi)$ is a positive definite hermitian form on $V \times V$.

Now we restrict our attention to genuine unitary supercuspidals of $\bar{G}$. Given such a $\pi$, when we restrict $\pi$ to $\overline{G^{*}}$ we obtain 4 inequivalent irreducible genuine unitary representations of $\overline{G^{*}}$. Indeed, $\left[\bar{G}: \overline{G^{*}}\right]=4$ and if $\pi$ is an irreducible representation of $\overline{G^{*}}$ occurring in this restriction with central character $\omega$, then $\pi$ conjugated by $\left(\begin{array}{ll}1 & 0 \\ 0 & a\end{array}\right), a \in \Sigma$, has central character $\chi_{a} \omega$. Moreover, we can recover $\pi$ by inducing up 
from any one of these. Thus we need only show the following:

Proposition 4.2. Fix a nontrivial $\psi$. Any irreducible admissible genuine unitary supercuspidal representation of $\overline{G^{*}}$ can be written as $\pi_{a q_{3}, \tilde{\sigma}, \lambda}^{\psi}$ for some $a \in k^{*}, \sigma \neq 1$, and unitary character $\lambda \in \widehat{k_{\sigma}^{*}}$.

Proof. We use the general Mackey theory of unitary representations of group extensions as found in Theorems 2 and 3 of Chapter III of [10]. In this notation, if $E$ is a group, $\hat{E}$ is the set of all irreducible unitary representations of $E$ and if $E$ is normal in $F, F$ acts on $\hat{E}$ by conjugation. Theorem 2 gives a description of $\widehat{G^{*}}$. Choose $\gamma \in \widehat{\mathrm{SL}}_{2}$, one from each $\overline{G^{*}}$-orbit. Set $\overline{G_{\gamma}^{*}}$ equal to the stability group of $\gamma$ and set

$$
\overline{G_{\gamma}^{*}} \sim=\left\{\nu \in \widehat{G_{\gamma}^{*}}: \nu \mid \overline{\mathrm{SL}}_{2} \text { is a multiple of } \gamma\right\} \text {. }
$$

For $\nu \in \overline{G_{\gamma}^{*}} \sim, \pi_{\nu}=\operatorname{Ind} \overline{G_{\gamma}^{*} \uparrow} \overline{G^{*}} \nu$ is irreducible and $\widehat{G^{*}}$ is the disjoint union

$$
\widehat{G^{*}}=\underset{\widehat{\mathrm{SL}}_{2} / \overline{G^{*}}}{\bigcup}\left\{\pi_{\nu}: \nu \in \overline{G_{\gamma}^{*}} \sim\right. \text {. }
$$

It can be shown that if $\pi=\pi_{\nu}$ is genuine supercuspidal, then so is $\nu$ and hence so is $\gamma$. Thus to compute all of the genuine (unitary) supercuspidals of $\overline{G^{*}}$ we need only compute $\overline{G_{\gamma}^{*}} \sim$ for $\gamma$ a genuine supercuspidal representation of $\overline{\mathrm{SL}}_{2}$ and induce up.

So, let $\gamma$ be such a representation. By Theorem 2.2(c), $\gamma$ is a $\pi_{a q_{3}, \tilde{\sigma}}^{\psi}$ for some $a \in k^{*}$ and $\sigma \neq 1$. Moreover, we can fix a unitary character $\chi_{0} \in \widehat{k}_{\sigma}^{*}$ and extend $\pi_{a q_{3}, \tilde{\sigma}}^{\psi}$ as in $\S 2$ to get a unitary representation of $\overline{G^{*}}$. This implies $\overline{G_{\gamma}^{*}}=\overline{G^{*}}$ and $\pi_{\nu}=\nu$ for all $\nu \in \overline{G_{\gamma}^{*}} \sim$. We now appeal to Theorem 3 which describes $\overline{G_{\gamma}^{*}} \sim$; it implies that any such $\nu$ is of the form $\lambda \otimes \pi_{a q_{3}, \tilde{\sigma}, \chi_{0}}^{\psi}$, where $\lambda$ is a unitary character of

$$
\overline{G^{*}} / \overline{\mathrm{SL}}_{2} \simeq\left\{\left(\begin{array}{cc}
1 & 0 \\
0 & a^{2}
\end{array}\right): a \in k^{*}\right\} \text {. }
$$

Thus

$$
\nu \simeq \pi_{a q_{3}, \tilde{\sigma}, \chi_{0} \lambda^{\prime}}^{\psi}, \quad \text { where } \lambda^{\prime}(a)=\lambda\left(\begin{array}{cc}
1 & 0 \\
0 & a^{2}
\end{array}\right)
$$

and the proof of Proposition 4.2 is complete.

REMARK 4.3. The theorems in [10] are stated for representations acting on a Hilbert space, but this causes no difficulty since we can apply Remark 2.3 .

Having constructed all of the irreducible genuine suercuspidals of $\bar{G}$ we obtain a simple proof of the following.

PROPOSITION 4.4. If $\pi$ is an irreducible admissible genuine supercuspidal representation of $\bar{G}$, then $\pi$ is not class one, i.e., contains no vector fixed by $K=\mathrm{GL}_{2}\left(O_{k}\right)$.

Before proving this, we need the following lemma.

LEMMA 4.5. Let $(Q, E)$ be an anisotropic quadratic form. Given a fractional ideal $\alpha$ of $k$, set $L=\{X \in E: Q(X) \in \alpha\}$. Then $X, Y \in L$ implies $B(X, Y) \in \alpha$.

Proof. It suffices to show $L$ is closed under addition. But this is done in the proof of 91.1 of [12]. 
Proof of Proposition 4.4. Fix a nontrivial $\psi$ with conductor $O_{k}$. Write $\pi$ as Ind $\pi_{q_{3}, \tilde{\sigma}, \lambda}$ with $\sigma$ nontrivial. It is convenient here not to use the representation space $\bigoplus_{i=1}^{4} \delta\left(k_{q_{3}, \tilde{\sigma}}^{*}\right)$ constructed at the end of $\S 2$, but rather $\bigoplus_{i=1}^{4} \delta_{\tilde{\sigma}}\left(H_{0}, V_{\tilde{\sigma}}\right)$ with the formulas in the matrices in Remark 2.10 defined as in formulas (2.4)-(2.6), (2.8).

Now suppose there was a

$$
\varphi=\left[\begin{array}{c}
\varphi_{1} \\
\vdots \\
\varphi_{4}
\end{array}\right]
$$

invariant under $K$. In particular $\pi\left(\begin{array}{ll}1 & 1 \\ 0 & 1\end{array}\right) \varphi=\varphi$ and so

$$
\begin{aligned}
\psi\left(q_{3}(X)\right) \varphi_{1}(X) & =\varphi_{1}(X), \\
\psi\left(\varepsilon q_{3}(X)\right) \varphi_{2}(X) & =\varphi_{2}(X), \\
\psi\left(\tilde{\omega} q_{3}(X)\right) \varphi_{3}(X) & =\varphi_{3}(X), \\
\psi\left(\varepsilon \tilde{\omega} q_{3}(X)\right) \varphi_{4}(X) & =\varphi_{4}(X) .
\end{aligned}
$$

If we set $L_{1}=\left\{X \in E: q_{3}(X) \in O_{k}\right\}$ and $L_{2}=\left\{X \in E: q_{3}(X) \in \tilde{\omega}^{-1} O_{k}\right\}$, then $\operatorname{supp} \varphi_{1}$ and $\operatorname{supp} \varphi_{2}$ are contained in $L_{1}$ while $\operatorname{supp} \varphi_{3}$ and $\operatorname{supp} \varphi_{4}$ are contained in $L_{2}$.

Now $\varphi K$ invariant implies $\pi\left(\begin{array}{rr}0 & 1 \\ -1 & 0\end{array}\right) \varphi=\varphi$, that is

$$
\begin{aligned}
\gamma_{\psi}\left(q_{3}\right) \hat{\varphi}_{1}(X) & =\varphi_{1}(X), \\
\gamma_{\psi}\left(\varepsilon q_{3}\right) \hat{\varphi}_{2}(X) & =\varphi_{2}(X), \\
\gamma_{\psi}\left(\tilde{\omega} q_{3}\right) \hat{\varphi}_{3}(X) & =\varphi_{3}(X), \\
\gamma_{\psi}\left(\varepsilon \tilde{\omega} q_{3}\right) \hat{\varphi}_{4}(X) & =\varphi_{4}(X) .
\end{aligned}
$$

For $\varphi_{1}$ this means $\gamma_{\psi}\left(q_{3}\right) \int_{H_{0}} \varphi_{1}(Y) \psi(B(X, Y)) d Y=\varphi_{1}(X)$. But since supp $\varphi_{1} \subset$. $L_{1}$,

$$
\gamma_{\psi}\left(q_{3}\right) \int_{L_{1}} \varphi_{1}(Y) \psi(B(X, Y)) d Y=\varphi_{1}(X)
$$

Since we need only consider $X$ in $L_{1}$, Lemma 5.2 implies $B(X, Y) \in O_{k}$ and so $\psi(B(X, Y)) \equiv 1$. Therefore

$$
\varphi_{1}(X)=\gamma_{\psi}\left(q_{3}\right) \int_{L_{1}} \varphi_{1}(Y) d Y=v_{1}
$$

for some vector $v_{1}$ in $V_{\tilde{\sigma}}$, when $X \in L_{1}$.

Similarly one concludes $\varphi_{2}(X)=v_{2}$ for $X \in L_{1}$ and $\varphi_{3}(X)=v_{3}, \varphi_{4}(X)=v_{4}$ for $X \in L_{2}$. Thus

$$
\varphi(X)=\left[\begin{array}{l}
v_{1} \\
v_{2} \\
v_{3} \\
v_{4}
\end{array}\right], \quad X \in L_{1} \cap L_{2}=L_{1}
$$


But each $\varphi_{i}$ is in $\delta_{\tilde{\sigma}}\left(H_{0}, V_{\tilde{\sigma}}\right)$; therefore $\varphi_{i}(g X)=\tilde{\sigma}(g) \varphi_{i}(X), g \in O\left(q_{3}\right), x \in H_{0}$. Furthermore $X \in L_{1} \cap L_{2}$ implies $g X \in L_{1} \cap L_{2}$, for $g$ in $O\left(q_{3}\right)$, and so $v_{i}=$ $\tilde{\sigma}(g) v_{i}, g \in O\left(q_{3}\right), i=1,2,3,4$. This is impossible unless $v_{i}=0$ since $\tilde{\sigma}$ is nontrivial. Thus $\varphi=0$ and $\pi$ is not class one.

5. Shimura correspondence. In Chapter 5 of [3] an injective correspondence between the genuine principal series representations of $\bar{G}$ and certain principal series representations of $G$ is given. The map is given by squaring the quasi-characters, i.e.

$$
S\left(\bar{\pi}\left(\mu_{1}, \mu_{2}\right)\right)=\pi\left(\mu_{1}^{2}, \mu_{2}^{2}\right),
$$

the right-hand side a representation of $G$ in the notation of [9].

We would like in this section to conjecture a correspondence, call it $S$ again, defined on the square-integrable genuine representations of $\bar{G}$.

Recall from [9] that one can associate an irreducible representation $\pi(\Omega)$ of $\mathrm{GL}_{2}(k)$ to any irreducible representation $\Omega$ of $H^{*}$. Moreover, if $\operatorname{dim} \sigma>1$, then $\pi(\Omega)$ is supercuspidal, and if $\Omega(h)=\chi(h \bar{h})$ for a quasi-character $\chi$ of $k^{*}$, then $\pi(\Omega)=\pi\left(\chi||^{1 / 2},\left.\chi\right|^{-1 / 2}\right)$. Also, the map $\Omega \rightarrow \pi(\Omega)$ is a bijection between the set of irreducible representations of $H^{*}$ and the set of supercuspidal and special representations of $G$.

Now consider $\pi=$ Ind $\pi_{q_{3}, \tilde{x}_{b}, x_{b} \lambda}$, for $b \notin k^{* 2}$. Proposition 3.3(a) implies $\pi \approx$ Ind $\pi_{q_{3}, \tilde{x}_{c}, \chi_{c} \lambda}$ for two other choices of $c\left(\bmod k^{* 2}\right)$ and that these are the only ways to write $\pi \approx R_{\lambda}$ with $\lambda(-1)=-1$. This suggests we make the following definition for $S$ :

$$
S\left(\operatorname{Ind} \pi_{q_{3}, \tilde{\sigma}, \lambda}\right)=\pi(\lambda \otimes \sigma) \approx \lambda \otimes \pi(\sigma) .
$$

For the above case we see that $S$ is well defined, and

$$
\begin{aligned}
S\left(R_{\lambda}\right) & =\pi\left(\chi_{c} \lambda \otimes \chi_{c}\right) \quad \text { any } c \notin k^{* 2} \\
& =\pi(\lambda)=\pi\left(\lambda||^{1 / 2}, \lambda||^{-1 / 2}\right), \quad \lambda(-1)=-1 .
\end{aligned}
$$

For $\sigma \equiv 1$ it is again easy to show that Ind $\pi_{q_{3}, \tilde{\sigma}, \lambda} \approx$ Ind $\pi_{q_{3}, \tilde{\sigma}_{1}, \lambda_{1}}$ implies $\lambda_{1}=\lambda$ and $\sigma_{1} \equiv 1$ Thus $S$ is well defined in this case also, and

$$
S\left(\text { Ind } \pi_{q_{3}, 0, \lambda}\right)=\pi\left(\lambda||^{1 / 2}, \lambda||^{-1 / 2}\right), \quad \lambda(-1)=1 .
$$

Note that this agrees with squaring the quasi-characters; indeed by Proposition 3.3(c),

$$
\pi=\text { Ind } \pi_{q_{3}, \tilde{\sigma}, \lambda} \approx \pi\left(\lambda^{1 / 2}||^{1 / 4}, \lambda^{1 / 2}||^{-1 / 4}\right) .
$$

Thus $S(\pi)=\pi\left(\lambda||^{1 / 2}, \lambda||^{-1 / 2}\right)$ as expected.

The problem in defining $S$ this way comes in the cases when $\operatorname{dim} \sigma>1$. We make the following conjecture:

Conjecture 5.1. If $b \in k^{* 2}$, or $b \in e_{1} e_{2} k^{* 2}$ with $e_{1}$ and $e_{2}$ as in Theorem 3.1, then

$$
\pi_{q_{3}, \chi_{b} \tilde{\otimes} \sigma}^{\psi} \approx \pi_{b q_{3}, \tilde{\sigma}}^{\psi}
$$


REMARK 5.2. The two choices of $b$ above are forced upon us. Indeed $\pi_{q_{3}, \tilde{\sigma}_{1}}^{\psi} \approx$ $\pi_{b q_{3}, \tilde{\sigma}_{2}}^{\psi}$ implies $C_{q_{3}, \tilde{\sigma}_{1}}=C_{b q_{3}, \tilde{\sigma}_{2}}=b C_{q_{3}, \tilde{\sigma}_{2}}$. Using the tables following Corollary 1.12 we see that this forces $\alpha\left(\sigma_{1}\right)=\alpha\left(\sigma_{2}\right)=\alpha$, and $b$ congruent to 1 or $e_{1} e_{2}$, where $e_{1}$ and $e_{2}$ are such that $\left(\alpha,-e_{i}\right)=-1$.

Corollary 5.3 (to Conjecture 5.1). For $\operatorname{dim} \sigma>1$, the map $S$ is well defined, injective, and onto the set of irreducible supercuspidal representations of $\mathrm{GL}_{2}(k)$ with square central character.

Proof. If Ind $\pi_{q_{3}, \tilde{\sigma}_{1}, \lambda_{1}} \approx$ Ind $\pi_{q_{3}, \tilde{\sigma}_{2}, \lambda_{2}}$, then, fixing $\psi$,

$$
\pi_{q_{3}, \tilde{\sigma}_{1}, \lambda_{1}}^{\psi} \approx\left(\pi_{q_{3}, \tilde{\sigma}_{2}, \lambda_{2}}^{\psi}\right)^{\left(\begin{array}{c}
1 \\
0 \\
b
\end{array}\right)} \approx \pi_{b q_{3}, \tilde{\sigma}_{2}, \lambda_{2}}^{\psi}
$$

for some $b \in \Sigma$. Comparing central characters gives $\lambda_{2}=\chi_{b} \lambda_{1}$ and restricting to $\overline{\mathrm{SL}}_{2}(k)$ gives $\pi_{q_{3}, \tilde{\sigma}_{1}}^{\psi} \approx \pi_{b q_{3}, \tilde{\sigma}_{2}}^{\psi}$ which, by Conjecture 5.1 and Theorem 2.2(a), implies $\sigma_{2} \approx \chi_{b} \otimes \sigma_{1}$. Thus $\lambda_{1} \otimes \sigma_{1} \approx \lambda_{2} \otimes \sigma_{2}$ and $S$ is well defined.

Now suppose

$$
S\left(\text { Ind } \pi_{q_{3}, \tilde{\sigma}_{1}, \lambda_{1}}\right) \approx S\left(\text { Ind } \pi_{q_{3}, \tilde{\sigma}_{2}, \lambda_{2}}\right) .
$$

Then $\pi\left(\lambda_{1} \otimes \sigma_{1}\right) \approx \pi\left(\lambda_{2} \otimes \sigma_{2}\right)$ which implies $\lambda_{1} \otimes \sigma_{1} \approx \lambda_{2} \otimes \sigma_{2}$. Comparing central characters gives $\lambda_{1}^{2}=\lambda_{2}^{2}$, i.e. $\lambda_{1}=\chi_{b} \lambda_{2}$ for some $b$. As a result $\sigma_{1} \approx \chi_{b} \otimes \sigma_{2}$. Corollary 1.12 implies $b \in k^{* 2}$ or $b \in e_{1} e_{2} k^{* 2}$, where $\left(\alpha\left(\sigma_{2}\right),-e_{i}\right)=-1$. Moreover Conjecture 5.1 implies

$$
\pi_{q_{3}, \tilde{\sigma}_{1}}^{\psi} \approx \pi_{q_{3}, \chi_{b} \tilde{\otimes} \sigma_{2}}^{\psi} \approx \pi_{b q_{3}, \tilde{\sigma}_{2}}^{\psi},
$$

and thus

$$
\pi_{q_{3}, \tilde{\sigma}_{1}, \lambda_{1}}^{\psi} \approx\left(\pi_{q_{3}, \tilde{\sigma}_{2}, \lambda_{2}}^{\psi}\right)^{\left(\begin{array}{cc}
1 & 0 \\
0
\end{array}\right)}
$$

Therefore Ind $\pi_{q_{3}, \tilde{\sigma}_{1}, \lambda_{1}} \approx$ Ind $\pi_{q_{3}, \tilde{\sigma}_{2}, \lambda_{2}}$ and $S$ is injective.

Finally suppose $\pi(\tau)$ has central character $\omega^{2}$. By Theorem 4.3(v) of [9], $\tau(a)=$ $\omega^{2}(a) I$ for $a \in k^{*}$. Let $\sigma=\omega^{-1} \otimes \tau$. $\sigma$ is an irreducible representation of $H^{*} / k^{*}$. If $\omega \in \widehat{k_{\sigma}^{*}}$, let $\pi=$ Ind $\pi_{q_{3}, \tilde{\sigma}, \omega}$. Then $S(\pi)=\pi(\tau)$. If $\omega \notin \widehat{k_{\tilde{\sigma}}^{*}}$ we can choose $c$ not congruent to 1 or $e_{1} e_{2}$, and, using Corollary 1.10 , verify that $\chi_{c} \omega \in \widehat{k_{c}^{*}} \tilde{\otimes}_{\sigma}$. Set

$$
\pi=\text { Ind } \pi_{q_{3}, \chi_{c}} \tilde{\otimes} \sigma, \chi_{c} \omega .
$$

Then $S(\pi)=\pi(\tau)$ and $S$ is surjective.

Suppose $F$ is a number field. For each place $v$ of $F$, set $\bar{G}_{v}=\overline{\mathrm{GL}}_{2}\left(F_{v}\right)$ and $G_{v}=\mathrm{GL}_{2}\left(F_{v}\right)$. In [6], $L$ - and $\varepsilon$-factors for representations of $\bar{G}_{v}$ are defined and an injective correspondence from unitary cuspidal representations of $\overline{G L}_{2}\left(A_{F}\right)$ to automorphic representations of $\mathrm{GL}_{2}\left(\mathbf{A}_{F}\right)$ is constructed. The correspondence is built up from local correspondences defined by matching $L$ - and $\varepsilon$-factors for the representations of $\bar{G}_{v}$ and $G_{v}$ (cf. $\S 7,14$ of [6]). It is our contention that $S$ is the local piece (when $k=F_{v}$ has odd residual characteristic) of this global correspondence. For $\pi=$ Ind $\pi_{q_{3}, \tilde{x}_{b}, \lambda}, b \in \Sigma, S$ does agree with the cases explicitly worked out in $\$ 6$ of [6]. For $\pi=$ Ind $\pi_{q_{3}, \tilde{\sigma}, \lambda}, \operatorname{dim} \sigma>1$, we observe that their central characters satisfy the relation:

$$
\omega_{S(\pi)}(a)=\omega_{\pi}\left(a^{2}\right), \quad a \in k^{*} .
$$


The results of [6] imply we need only relate the $\varepsilon$-factors of $\pi$ and $S(\pi)$, the $L$-factors both being $\equiv 1$. Although we have computed the $\varepsilon$-factors for such $\pi$, the relation to the $\varepsilon$-factor for $S(\pi)=\pi(\lambda \otimes \sigma)$ is not yet apparent.

REMARK 5.4. In [2], the image of the global correspondence is characterized using the trace formulas for $\overline{\mathrm{GL}}_{2}(\mathrm{~A})$ and $\mathrm{GL}_{2}(\mathrm{~A})$. In particular, it is shown that the image of the local correspondence restricted to the supercuspidal representations not equivalent to $R_{\lambda}, \lambda(-1)=-1$, is precisely as indicated in Corollary 5.3.

Finally, a few words about how one might establish Conjecture 5.1. One approach is to use the computation of $\varepsilon$-factors to deduce the equivalence of Ind $\pi_{q_{3}, \tilde{\sigma}, \lambda}$ and Ind $\pi_{q_{3}, \chi_{b} \tilde{\otimes} \sigma, \chi_{b} \lambda},\left(b \in e_{1} e_{2} k^{* 2}\right)$. This implies

$$
\pi_{q_{3}, x_{b} \tilde{\otimes} \sigma, x_{b} \lambda}^{\psi} \approx\left(\pi_{q_{3}, \tilde{\sigma}, \lambda}^{\psi}\right)^{\left(\begin{array}{cc}
1 & 0 \\
0
\end{array}\right)} \approx \pi_{b q_{3}, \tilde{\sigma}, \lambda}^{\psi}
$$

and we obtain the conjecture. This would involve a detailed analysis of the functions $j_{q_{3}, \tilde{\sigma}}$ and $j_{q_{3}, \chi_{b}} \tilde{\otimes} \boldsymbol{\sigma}$, where

$$
j_{Q, \tau}(Q(X), Q(Y))=\int_{O(Q)}\left(\tau(g) \psi(B(g X, Y)) v_{X}, v_{Y}\right) d g
$$

since these appear in the expression for the $\varepsilon$-factors.

A second approach, suggested by Howe, is to use the lattice model for the Weil representation to study the correspondence $\tilde{\sigma} \rightarrow \pi_{c q_{3}, \tilde{\sigma}}^{\psi}$ in more detail. The representations of $\overline{\mathrm{SL}}_{2}(k)$ are induced from representations of $\mathrm{SL}_{2}\left(O_{k}\right)$ and one should be able to match these $\mathrm{SL}_{2}\left(O_{k}\right)$-representations with $O\left(c q_{3}\right)$-representations in such a way as to make the equivalences among the Weil representations easier to find.

\section{BIBLIOGRAPHY}

1. H. Boerner, Representations of groups, 2nd ed., North-Holland, Amsterdam, 1970.

2. Y. Flicker, Automorphic forms on covering groups of $\mathrm{GL}_{2}$, Invent. Math. 57 (1980), 119-182.

3. S. Gelbart, Weil's representation and the spectrum of the metoplectic group, Lecture Notes in Math., vol. 530, Springer-Verlag, Berlin and New York, 1976.

4. S. Gelbart and I. I. Piatetski-Shapiro, Automorphic L-functions of half-integral weight, Proc. Nat. Acad. Sci. U.S.A. 75 (1978), 1620-1623.

5. __ Distinguished representations and modular forms of half-integral weight, Invent. Math. 59 (1980), 145-188.

6. _ On Shimura's correspondence for modular forms of half-integral weight, Proc. Colloq. on Automorphic Forms, Representation Theory and Arithmetic (Bombay, 1979).

7. S. Gelbart, I. I. Piatetski-Shapiro and R. Howe, Uniqueness and existence of Whittaker models for the metaplectic group, Israel J. Math. 34 (1979), 21-37.

8. I. M. Gelfand and M. I. Graev, Representations of quaternion groups over locally compact and function fields, Functional Anal. Appl. 2 (1968).

9. H. Jacquet and R. P. Langlands, Automorphic forms on GL(2), Lecture Notes in Math., vol. 114, Springer-Verlag, Berlin and New York, 1970.

10. R. Lipsman, Group representations, Lecture Notes in Math., vol. 388, Springer-Verlag, Berlin and New York, 1974.

11. C. Moen, Ph. D. Thesis, University of Chicago, 1979.

12. T. O’Meara, Quadratic forms, 2nd ed., Springer-Verlag, Berlin and New York, 1971.

13. S. Rallis and G. Schiffman, Distributions invariantes pour la groupe orthogonal, Analyse Harmonique sur les Groupes de Lie, Lecture Notes in Math., vol. 497, Springer-Verlag, Berlin and New York, 1975.

14. __ Representations supercuspidales du groupe metaplectique, J. Math. Kyoto Univ. 17 (1977).

15. J. P. Serre, A course in arithmetic, Springer-Verlag, New York, 1973.

Current address: 11 Wisteria Drive, Apt. 3J, Fords, New Jersey 08863 